\title{
The volume densities of giant molecular clouds in $\mathrm{M} 83^{\star, \star \star}$
}

\author{
J. S. Heiner ${ }^{1,2}$, R. J. Allen ${ }^{2}$, O. I. Wong ${ }^{3}$, and P. C. van der Kruit ${ }^{1}$
}

\author{
${ }^{1}$ Kapteyn Astronomical Institute, University of Groningen, PO Box 800, 9700 AV Groningen, The Netherlands \\ e-mail: heiner@astro.rug.nl \\ 2 Space Telescope Science Institute, Baltimore, MD 21218, USA \\ 3 Astronomy Department, Yale University, PO Box 208101, New Haven, CT 06520-8101, USA
}

Received 19 June 2008 / Accepted 24 July 2008

ABSTRACT

\begin{abstract}
Using observed GALEX far-ultraviolet (FUV) fluxes and VLA images of the 21-cm HI column densities, along with estimates of the local dust abundances, we measure the volume densities of a sample of actively star-forming giant molecular clouds (GMCs) in the nearby spiral galaxy M 83 on a typical resolution scale of $170 \mathrm{pc}$.

Our approach is based on an equilibrium model for the cycle of molecular hydrogen formation on dust grains and photodissociation under the influence of the FUV radiation on the cloud surfaces of GMCs.

We find a range of total volume densities on the surface of GMCs in M 83, namely $0.1-400 \mathrm{~cm}^{-3}$ inside $R_{25}, 0.5-50 \mathrm{~cm}^{-3} \mathrm{outside}_{25}$. Our data include a number of GMCs in the HI ring surrounding this galaxy. Finally, we discuss the effects of observational selection, which may bias our results.
\end{abstract}

Key words. galaxies: individual: M 83 - galaxies: ISM - ISM: clouds - ISM: molecules - ultraviolet: galaxies - radio lines: galaxies

\section{Introduction}

This paper aims to measure the total gas densities in a sample of giant molecular clouds (GMCs) in the nearby spiral M 83 (NGC 5236), using a method initially proposed by Allen et al. (1997). This method is based on the simple (but unavoidable) fact that ultraviolet photons from young, newly-formed stars will react back on the surrounding parent GMCs, dissociating the molecular gas on their surfaces and turning the (virtually invisible) $\mathrm{H}_{2}$ into its easily-detected atomic form. The motivation for this approach was provided by the discovery of Allen et al. (1986) that the HI delineating the spiral arms in a nearby galaxy showed a large-scale morphology that was more consistent with photodissociation near the HII regions than it was with compression of the HI farther upstream in the spiral shock. The presence of atomic hydrogen is then indicative of a photodissociation region (PDR). The method was first applied in some detail to M101 by Smith et al. (2000) and more recently to M 81 by Heiner et al. (2008).

The large, nearby spiral M 83 has been a frequent target of searches for molecular gas using the $\mathrm{CO}(1-0)$ spectral line, which is easily detectable in M 83. However, such studies have only recently begun to be carried out with sufficient linear (spatial) resolution to discern differences in the location of molecular gas and the emissions from young, hot stars. For example, Lord \& Kenney (1991) describe the presence of molecular (CO) emission $300 \mathrm{pc}$ downstream from M 83's eastern spiral arm dust lane, detected with a $5^{\prime \prime} \times 12^{\prime \prime}$ beam. Rand et al. (1999) find that the CO emission is spatially separated from the dust lane as well as the young stars on scales of a few hundred parsec. They

\footnotetext{
* Full Tables 1 and 3 are only available in electronic form at the CDS via anonymous ftp to cdsarc.u-strasbg. fr $(130.79 .128 .5)$ or via http://cdsweb.u-strasbg.fr/cgi-bin/qcat?J/A+A/489/533

$\star \star$ Full Fig. 3 is only available in electronic form at

http://www . aanda.org
}

suggest UV heating, cosmic-ray heating or a two-component molecular phase to explain this morphology. Their observations show features that show similarity to the largest GMCs in the Milky Way with masses on the order of several millions of solar masses. Wall et al. (1993) explore the CO content in the central kiloparsec of M 83 and infer a low-density component $\left(n_{\mathrm{H}_{2}} \lesssim 10^{3}-10^{4} \mathrm{~cm}^{-3}\right)$ and a warm (above $50 \mathrm{~K}$ ), high-density component $\left(n_{\mathrm{H}_{2}} \gtrsim 10^{4}-10^{5} \mathrm{~cm}^{-3}\right)$. Crosthwaite et al. (2002) study $\mathrm{CO}(1-0), \mathrm{CO}(2-1)$ and neutral gas in $\mathrm{M} 83$, observing a strong truncation of the molecular disk at $6^{\prime}$ accompanied by a warped atomic outer disk. Using the conventional assumptions about how to convert $\mathrm{CO}$ surface brightness to $\mathrm{H}_{2}$ column density, they conclude that roughly $80 \%$ of the total gas mass in $\mathrm{M} 83$ is $\mathrm{H}_{2}$. The $\mathrm{HI} 21 \mathrm{~cm}$ and $\mathrm{CO}$ surface brightness are found to be correlated, but with a complex pattern of offsets. They speculate that the temperature of the $\mathrm{CO}$ gas is $>20 \mathrm{~K}$ in the nucleus and $<7 \mathrm{~K}$ in the outer disk. Lundgren et al. (2004) give a full overview of previous observations and present full $\mathrm{CO}(1-0)$ and $\mathrm{CO}(2-1)$ maps based on thousands of telescope pointings, but with a modest spatial resolution of $\sim 1 \mathrm{kpc}$. They conclude that the molecular gas spiral arms mostly trace the dust lanes. They expect the $\mathrm{H}_{2}$ mass to dominate that of $\mathrm{HI}$ within $7.3 \mathrm{kpc}$ of the center. At their linear resolution, $\mathrm{CO}$ and $\mathrm{HI}$ emissions are correlated strongly within the optical disk. Vogler et al. (2005) look into various tracers of star formation, including Polycyclic Aromatic Hydrocarbon (PAH) emission lines, another potential indicator of PDRs. Various combinations of these lines prove to be good tracers of star forming regions in M 83. They are found predominantly in the spiral arms. We studied the occurrence of PAHs near PDRs in M 81 (Heiner et al. 2008) previously and confirmed that they are found near the star forming regions in almost all cases. Similar M 83 data was not available at this time.

Thilker et al. (2005) find and discuss sites of recent star formation in the extreme outer disk of M 83, associated with the warped HI disk of M 83, which raises questions about star 
formation efficiency and modes of star formation. The nature of this outer disk is investigated further in Gil de Paz et al. (2007b), who find evidence that individual young stars are responsible for the UV emission in the outer regions. Zaritsky \& Christlein (2007) find that these UV sources are quite common out to $2 \times R_{25}$. In this paper we include candidate PDRs outside the main optical disk of M 83 in an effort to probe the amount of available gas in this environment.

The suspected high densities of molecular gas in M 83, the morphology of the gas in the spiral arms, and the evidence of recent star formation in the outer regions, make this galaxy an excellent target for a study of photodissociated atomic hydrogen and the volume densities of M 83's GMCs using our method.

In Sect. 2, we explain our approach and the data we used. In Sect. 3 we present our results. We discuss and summarize these results in Sect. 4.

\section{Method}

In this section we briefly describe the data and how we used it. The same method that we used in Heiner et al. (2008) has been applied here. For every candidate PDR, the far-UV flux, local HI column densities, dust-to-gas ratio and separation between the UV source and the surrounding HI features were determined to calculate the total hydrogen volume density.

The distance to M 83 is taken to be $4.5 \mathrm{Mpc}$, as measured from Cepheids by Thim et al. (2003). We used GALEX UV data of M 83, where the FUV and near-UV (NUV) images were matched for astrometric accuracy. The images have an estimated angular resolution of $8^{\prime \prime}$, which is a bit poorer than the generic GALEX resolution, since M 83 is on the edge of the GALEX field of view due to pointing constraints. We did not use the NUV image otherwise. We obtained the $21 \mathrm{~cm}$ HI data from the THINGS team (Walter et al. 2008, AJ, submitted). The radio beam size is $10.4 \times 5.6^{\prime \prime}$. The data were analyzed using the Groningen Image Processing System GIPSY (Ekers et al. 1973; van der Hulst et al. 1992; Vogelaar \& Terlouw 2001).

Although spatial completeness was not our aim, we attempted to select our candidate PDRs to be bright and isolated on the GALEX image and to have a uniform spread in galactocentric radius as well as FUV flux, in a similar way as was done in Heiner et al. (2008). First, GIPSY's POINTS routine was used to automatically detect FUV sources with a signal-to-noise of 20 or more. The other input parameters were kept at their default value. This yielded 86 sources $^{1}$. Using a histogram of the distribution of the sources with galactocentric radius, we randomly removed a small number of sources to even out the distribution. We also removed sources that matched detections in the HST Guide Star Catalog v2.2, see e.g. Lasker et al. (2008). We manually added some sources to improve the spread in galactocentric radius, selected by eye. These sources stood out visually, but we did not enforce a specific signal-to-noise ratio. Finally, these sources were automatically fitted with gaussians. In some two or three cases this led to visible confusion from nearby FUV sources as can be seen in the location plot (Fig. 1). The final source count was 76 . These sources are listed in Table 1.

We used the tilted ring model from Rogstad et al. (1974) to correct for a different position angle and inclination at larger radii. This deprojection correction changes the actual galactocentric radii of the candidate PDRs as well as the assumed deprojected separation between the UV source and its associated $\mathrm{HI}$ patches. This information was used in combination with the

1 This translates into a typical rms noise in the FUV flux of $1 \%$. metallicity data from Gil de Paz et al. (2007b) to obtain the dustto-gas ratio (see Issa et al. 1990). We used

$\log \delta / \delta_{0}=-0.051 R+0.43$,

where $\delta / \delta_{0}$ is the local dust-to-gas ratio scaled to the value in the solar neighborhood. We adopt a solar metallicity of 8.69 (Allende Prieto et al. 2001). The galactocentric radius $R$ is in kiloparsec. In subsequent plots we normalize $R$ with $R_{25}$, which is $7.63 \mathrm{kpc}$ ( or $\approx 6^{\prime}$ ) from Vila-Costas \& Edmunds (1992). We assume that this relation covers the full range of galactocentric radii we investigated. It becomes clear from the literature that the metallicity in the outer regions of M 83 is still uncertain and results have only recently become available. For this reason we explore a number of alternatives. We also considered, among others, Bresolin \& Kennicutt (2002). The different alternatives are plotted in Fig. 2. We have adopted the most recent high metallicity fit in Gil de Paz et al. (2007b) as our preferred model.

The (local background subtracted) FUV fluxes of the candidate PDRs were determined next. For each such region, the distances from the central source to the nearest HI patches was determined. An $\mathrm{HI}$ patch is defined as a local maximum in the HI column density. A background level of $2 \times 10^{20} \mathrm{~cm}^{-2}$ was subtracted within $0.9 R_{25}$ and $5 \times 10^{19} \mathrm{~cm}^{-2}$ outside this radius. These values are based on the general background level of HI emission surrounding M 83. We also measured HI background levels within the optical disk of M 83 as well as considered minimum column density values throughout M 83. This is an attempt to isolate the $\mathrm{HI}$ formed by dissociating radiation. Since the background level is close to the sensitivity limit, some measurements end up close to zero. The resulting values of $n$ are generally not very sensitive to varying the background HI column. Rewriting Eq. (2) to yield $n$ (see Eq. (4) in Heiner et al. 2008) shows that $n$ does not change much as long as the subtracted background column remains substantially lower than the scaling factor of $7.8 \times 10^{20} \mathrm{~cm}^{-2}$, as is the case here. We measured distances out to $22^{\prime \prime}$. Those distances were then used together with the FUV flux to calculate the incident flux impinging on each $\mathrm{HI}$ patch. A $1500 \AA$ foreground extinction correction of 0.52 mag based on Schlegel et al. (1998) and Gil de Paz et al. (2007a) was applied to the FUV fluxes. Two example sources are plotted in Fig. 3.

Finally, all these elements were used to calculate the total hydrogen volume density by inverting Eq. (A2) from Allen et al. (2004) and including a variable dust-to-gas ratio (cf. Eq. (6) in Allen 2004). The final equation to be inverted is:

$N_{\mathrm{HI}}=\frac{7.8 \times 10^{20}}{\delta / \delta_{0}} \ln \left[1+\frac{106 G_{0}}{n}\left(\frac{\delta}{\delta_{0}}\right)^{-1 / 2}\right] \mathrm{cm}^{-2}$

where $N_{\mathrm{HI}}$ is the (background subtracted) atomic hydrogen column density (in $\mathrm{cm}^{-2}$ ), $\delta / \delta_{0}$ is the dust-to-gas ratio scaled to the solar neighborhood value, $G_{0}$ is the incident flux measured at the HI patch (the same $G_{0}$ as used in e.g. Allen et al. (2004), Appendix B - see Heiner et al. (2008) for a more extended description of how this is measured) and $n$ is the total hydrogen volume density, where $n=n_{\mathrm{HI}}+2 n_{\mathrm{H}_{2}}\left(\mathrm{~cm}^{-3}\right)$. This gas is mostly atomic on the surface of the GMC and mostly molecular deep inside the cloud.

\section{Results}

The measured FUV fluxes are presented first, followed by the associated HI column densities. The derived incident fluxes $G_{0}$ are presented next, followed by the resulting total hydrogen volume densities. Finally, we deal with a number of selection effects. 


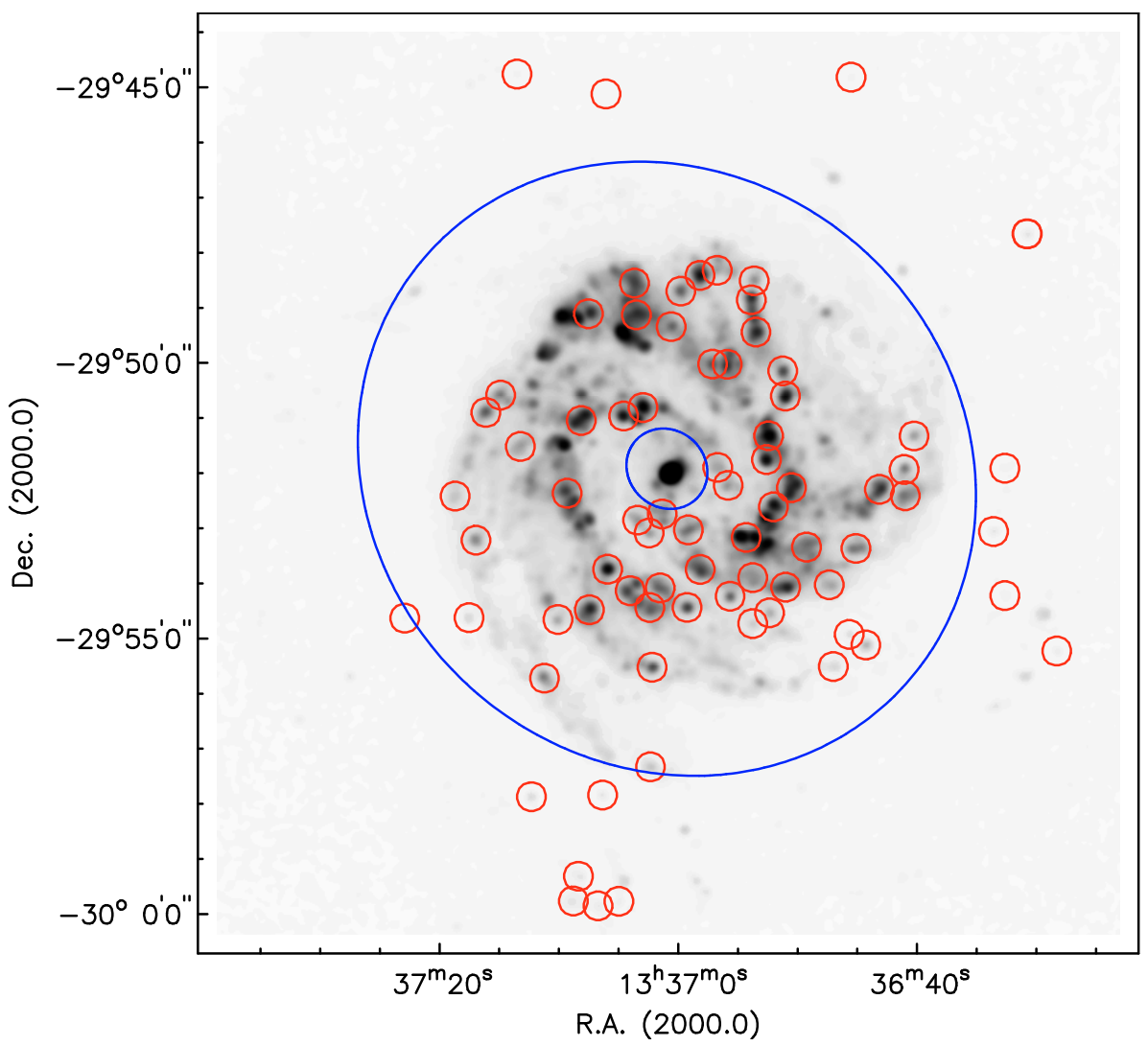

Fig. 1. The location of the candidate PDRs are shown with the GALEX FUV image in the background. Not all FUV sources are visible on this image, but all sources are sources of FUV radiation. The inner circle signifies a $1 \mathrm{kpc}$ galactocentric radius, the outer one signifies $R_{25}$.

Table 1. Locations and FUV fluxes of candidate PDRs. The full table is only available in electronic form at the CDS.

\begin{tabular}{cccccccc}
\hline \hline Source No. & RA (2000) & Dec (2000) & $\begin{array}{c}\text { Radius } \\
(\mathrm{kpc})\end{array}$ & $\begin{array}{c}F_{\mathrm{FuV}} \\
10^{-15} \mathrm{erg} \mathrm{cm}^{-2} \mathrm{~s}^{-1} \AA^{-1}\end{array}$ & $\begin{array}{c}\text { Aperture } \\
"\end{array}$ & $\begin{array}{c}\text { Grayscale start } \\
\left(10^{19} \mathrm{~cm}^{-2}\right)\end{array}$ & \begin{tabular}{c} 
Increment \\
\hline
\end{tabular} \\
\hline 1 & 133701.346 & -295244.72 & 1.1 & 5.16 & 19 & 1 & 1.35 \\
2 & 133656.700 & -295154.00 & 1.3 & 2.08 & 16 & 1 & 1.33 \\
3 & 133703.402 & -295251.43 & 1.5 & 2.20 & 14 & 1 & 1.37 \\
4 & 133655.827 & -295213.58 & 1.5 & 2.56 & 18 & 1 & 1.30 \\
5 & 133659.158 & -295302.08 & 1.6 & 6.83 & 21 & 1 & 1.36 \\
6 & 133702.955 & -295048.84 & 1.6 & 12.24 & 14 & 1 & 1.37 \\
7 & 133704.572 & -295058.09 & 1.6 & 6.89 & 12 & 1 & 1.33 \\
8 & 133702.445 & -295305.43 & 1.7 & 1.18 & 10 & 1 & 1.35 \\
9 & 133708.120 & -295102.97 & 2.4 & 20.81 & 20 & 1 & 1.37 \\
10 & 133654.317 & -295310.26 & 2.5 & 21.61 & 17 & 1 & 1.35 \\
\hline
\end{tabular}

The locations of our candidate PDRs are shown in Fig. 1 and tabulated in Table 1. We attempted to get a selection of sources representative in both their galactocentric radius as well as their UV flux, as was mentioned previously. The table also includes the aperture of each FUV source within which its flux was determined, the starting grayscale level in the detailed plots and the grayscale level increment, where each subsequent level is the previous level multiplied by the increment. While the starting grayscale level is usually $1 \times 10^{19} \mathrm{~cm}^{-2}$, it is different for some sources to enhance the grayscale contrast. (See Fig. 3 for two sample plots; the full set is available in the electronic edition of this Journal.)

The disk of M 83 is warped at larger galactocentric radii. This influences the deprojected galactocentric radius that we used to determine the dust-to-gas ratio. The distance from the FUV sources to the associated HI patches is also affected, since we use the position angle and declination to deproject the separation $\rho_{\mathrm{HI}}$ (see Heiner et al. 2008, for a more detailed description of the procedure and projection issues). Table 2 shows the values we adopted from the tilted ring model of Rogstad et al. (1974).
The background subtracted FUV flux of each source is plotted in Fig. 4 and listed in Table 1. The background UV level was determined at the radius listed in the aperture column. A range of fluxes can be seen, which seems to be decreasing towards the outer parts of M 83. However, crowding effects are a possible cause of this trend. This is indicated in Fig. 4. In addition we have made no attempt to obtain a statistically complete sample of sources. A detection limit is plotted based on the noise level of the FUV data and the method we used to select our sources. These fluxes have not been corrected for extinction. We did not select sources close to the central starburst (see e.g. Boissier et al. (2005) for a description of the UV radial profiles found by GALEX).

In an attempt to capture as much information as possible per candidate PDR, we determined the distance $\rho_{\mathrm{HI}}$ of every HI patch to its central UV source (Fig. 5). This means that every FUV source has a number of measurements associated with it, which appear in the plot as a number of points at the same galactocentric radius. At an arbitrary distance of about 480 pc from the FUV source (roughly 22") we stopped recording HI patches, 


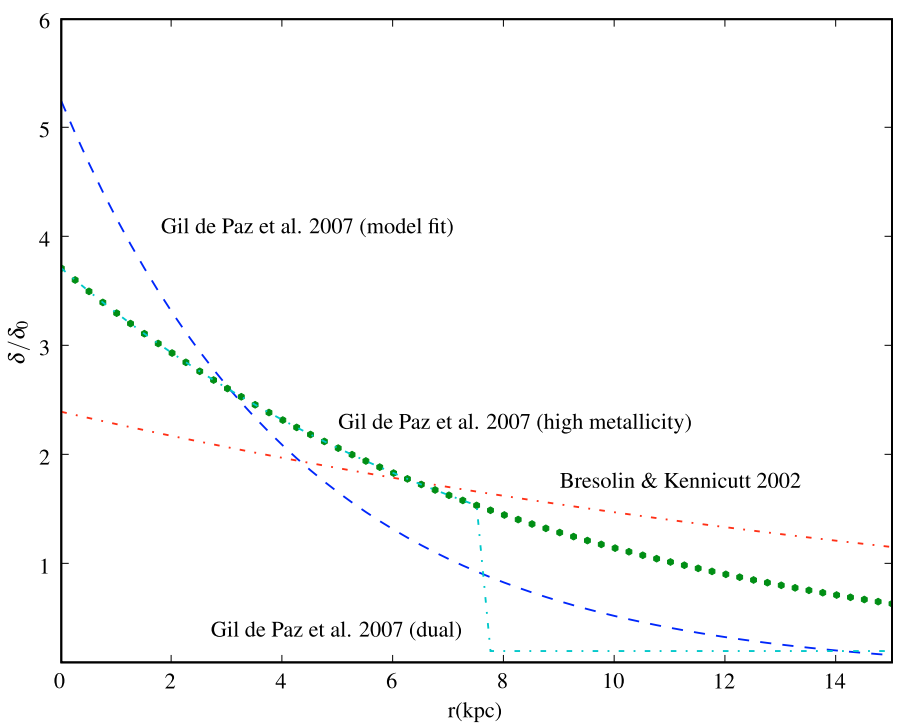

Fig. 2. Different possible dust-to-gas ratio models are plotted. There are three models suggested by Gil de Paz et al. (2007b) and Gil de Paz (private communication, 2008), namely their high metallicity fit (our default one), their fit based on PDR modeling and a slope based on the high metallicity fit inside $R_{25}$ combined with a constant (low) metallicity in the outer regions. We also considered the one based on Bresolin \& Kennicutt (2002).

since at that distance the association of these patches to that particular UV source becomes highly unlikely. This cut-off is arbitrary, since it becomes progressively less likely for an HI patch to be associated with the central UV source. The cut-off was chosen with the typical size of large scale PDRs in mind (a few hundred parsec) and is labeled "confusion limit" in the plot. Large scale PDRs are expected to span a few hundred parsec at most and other UV associations will also be encountered. The separations are quantized since we used $1^{\prime \prime}$ rings. In certain cases the candidate PDRs themselves are fairly close to each other. In such a case an HI patch can be attributed to two UV sources, but without further morphological information no distinction can be made. The minimum observable distance is determined by the resolution of our data and is about $30 \mathrm{pc}$. This plot also shows the coverage in galactocentric radius, which shows a few gaps where no FUV source was found (or selected) using the selection method described earlier. The full results are tabulated in Table 3, where the different HI patches per FUV source are labeled a, b, c, and so on. $\rho_{\mathrm{HI}}, N_{\mathrm{HI}}$, the incident flux $G_{0}$, the source contrast $G / G_{\mathrm{bg}}$, the total hydrogen volume density $n$, and the fractional errors are listed in this table.

In rare cases the HI column density is very low, where the measured column is close to the value of the subtracted background. No columns higher than $\approx 2.5 \times 10^{21} \mathrm{~cm}^{-2}$ were found. This is potentially a beam smoothing effect, where we only resolve HI patches partially. We conclude this from our work on M 33 (Heiner et al., in prep.). At higher linear resolution we observe higher HI columns, which points to better resolution of the HI clouds in M 33 .

The incident flux $G_{0}$ (corrected for foreground extinction) on each individual HI patch is plotted in Fig. 6. A set of points in the vertical direction shows all resulting $G_{0}$ for an individual UV source with multiple HI patches. The average $G_{0}$ declines going outward, with no distinct break at the edge of M 83's optical disk. A hint of an upturn at the location of M 83's HI ring is visible at the extreme right end of the plot. As $G_{0}$ is a
Table 2. Adopted tilted ring values from Rogstad et al. (1974).

\begin{tabular}{ccc}
\hline \hline Radius $\left({ }^{\prime}\right)$ & PA (degrees) & $i$ (degrees) \\
\hline 0.5 & 45 & 24 \\
1.0 & 45 & 24 \\
1.5 & 45 & 24 \\
2.0 & 45 & 24 \\
2.5 & 46 & 24 \\
3.0 & 46 & 24 \\
3.5 & 46 & 24 \\
4.0 & 46 & 25 \\
4.5 & 46 & 26 \\
5.0 & 49 & 27 \\
5.5 & 52 & 28 \\
6.0 & 59 & 30 \\
6.5 & 67 & 32 \\
7.0 & 75 & 34 \\
7.5 & 83 & 36 \\
8.0 & 91 & 37 \\
8.5 & 99 & 39 \\
9.0 & 106 & 40 \\
9.5 & 113 & 42 \\
10.0 & 120 & 43 \\
10.5 & 127 & 45 \\
11.0 & 133 & 46 \\
11.5 & 139 & 47 \\
12 & 145 & 49 \\
12.5 & 150 & 50 \\
\hline & &
\end{tabular}

combination of the FUV flux and the FUV source/HI patch separation, it is also influenced by the possible crowding effects that are indicated in Fig. 4. The minimum $G_{0}(0.005)$ is due to the maximum $\rho_{\mathrm{HI}}$ that we measure, combined with the lowest measured flux, and is therefore a sensitivity limit. The highest $G_{0}(565)$ that we could obtain is determined by the lowest measurable $\rho_{\mathrm{HI}}$ and the highest FUV flux. Both limits are plotted. The open circles are incident fluxes with a source contrast of less than 0.5 . This means that the incident source flux has $50 \%$ of the strength of the ambient FUV field incident on the HI patch or less. The HI corresponding to the closed circles can be assumed to have a higher chance of having been produced by photodissociating UV radiation, since the central UV source is dominant with respect to the background radiation field (the source contrast is higher). However, these highest source contrasts occur when the separation between the central UV source and the associated HI patch is the lowest - at close separation projection effects are more of a problem, which means a higher chance of underestimating the separation and overestimating the incident flux. A high source contrast at an HI patch therefore needs to be viewed with caution.

The (beam averaged) atomic hydrogen column density of each HI patch in our candidate PDRs is plotted in Fig. 7. A selection of local maxima are counted as HI patches. Near the center of M 83 there is little $\mathrm{HI}$ emission visible against the continuum source at the nucleus (e.g. Tilanus \& Allen 1993). The maximum observed column density rises to a broad peak around $0.5 R_{25}$. The end of M83's disk is clearly visible where the maximum HI column densities drop, but HI is still detected. Finally, there is a slight increase where M 83's HI ring is located.

An HI background value was subtracted as described previously, in an attempt to capture only the HI produced by the incident FUV radiation from the FUV source nearby. The resulting values of $n$ are relatively insensitive to varying the background HI column. Background-subtracted values below the sensitivity limit were discarded. 

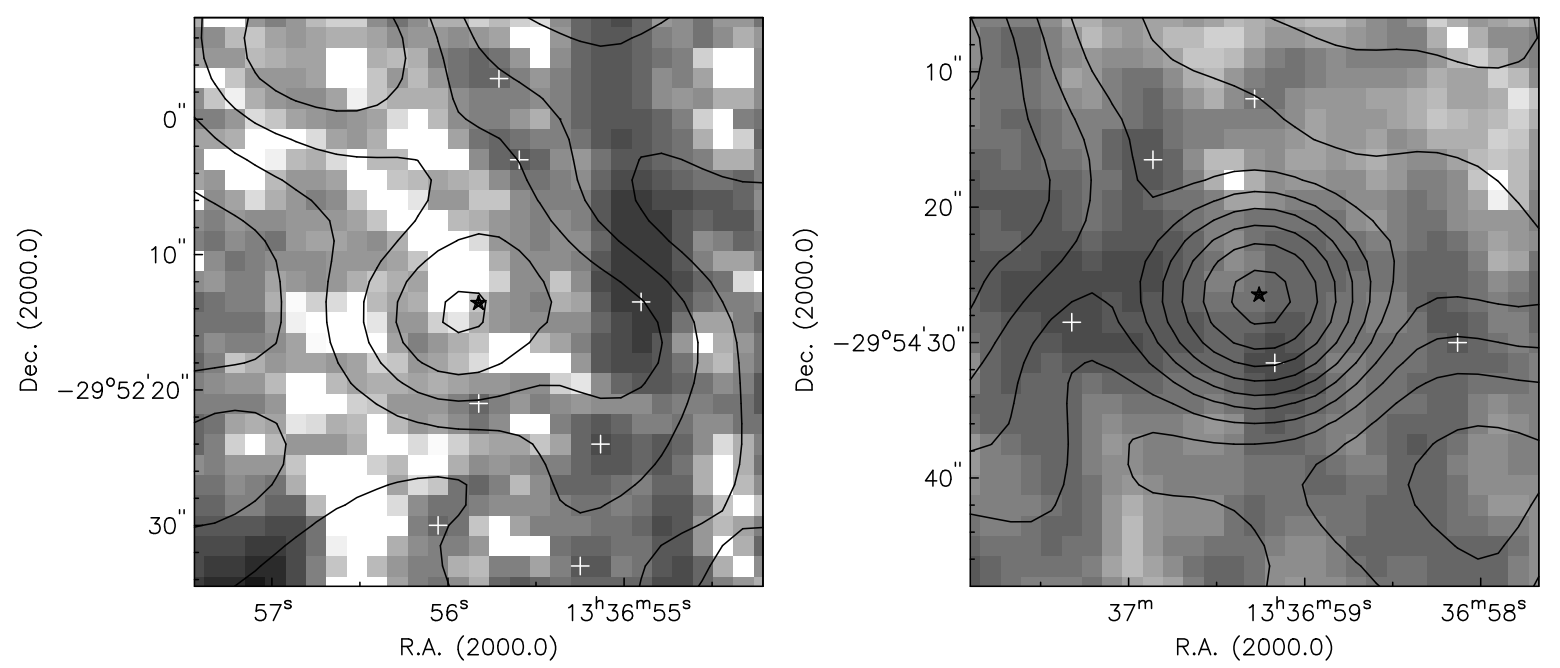

Fig. 3. Sources 4 (left panel) and 24 (right panel) are plotted as examples of candidate PDRs, showing HI grayscales and FUV contours. The location of the central source is marked with a black star, the locations of the HI patches are marked with white crosses. The FUV contours start at $7 \times 10^{-19} \mathrm{erg} \mathrm{cm}^{-2} \mathrm{~s}^{-1} \AA^{-1}$ and each consecutive level is 1.34 times the previous one. The HI grayscale levels are constructed similarly, see Table 1. Both sources show a central source surrounded by HI complexes, where the distribution is smoother in Source 24 . The full set of sources is available in the electronic version of this paper (without the white crosses).

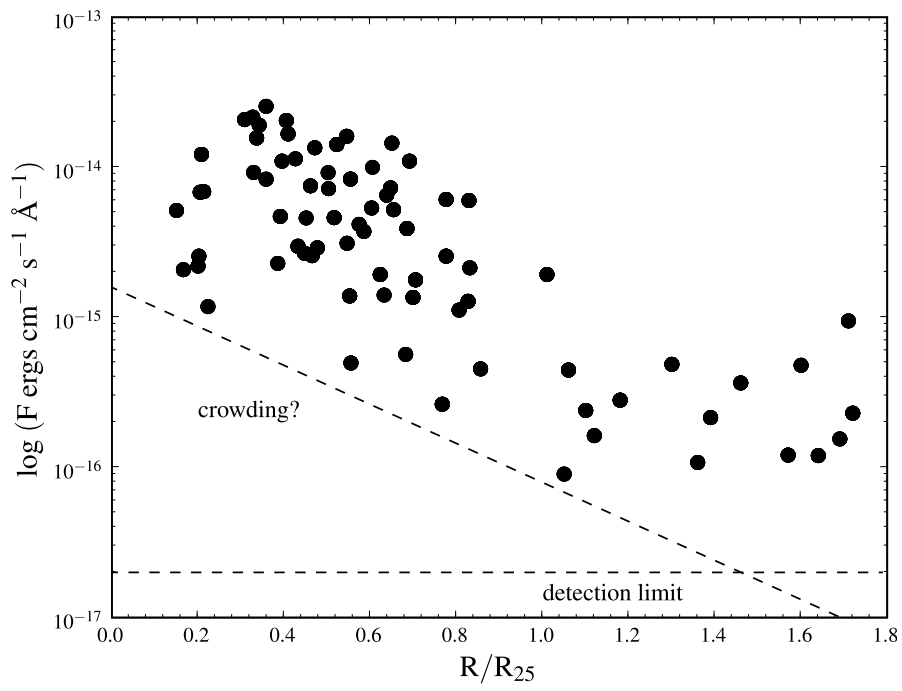

Fig. 4. The FUV fluxes of the candidate PDRs are plotted here on a logarithmic scale. Outside of $R_{25}$ the source fluxes seem to flatten out. A detection limit has been included, as well as a possible crowding limit. The angular resolution of the data is approximately $8^{\prime \prime}$.

The calculated total hydrogen volume density is shown in Fig. 8, based on the Gil de Paz et al. (2007b) high metallicity assumption, which is our preferred dust model. Values span a range of two orders of magnitude mostly, with values of a few thousand $\mathrm{cm}^{-3}$ in the inner parts of M 83. Outside M 83's optical disk the values of $n$ are generally lower. In this plot, again closed circles are plotted for HI patches that show a source contrast higher than 0.5 (meaning the source is half as bright as the UV background or stronger), and open circles for those with lower source contrast. The former may be considered more reliable than the latter, since the UV source is considered to be more clearly responsible for the dissociated HI. The FUV source contrasts $G / G_{\mathrm{bg}}$ are listed in Table 3 . The fractional errors in Table 3 are as calculated in the Heiner et al. (2008) M 81 results, except that we used a relative error for the dust-to-gas ratio of $20 \%$ instead of a constant error. The average fractional error

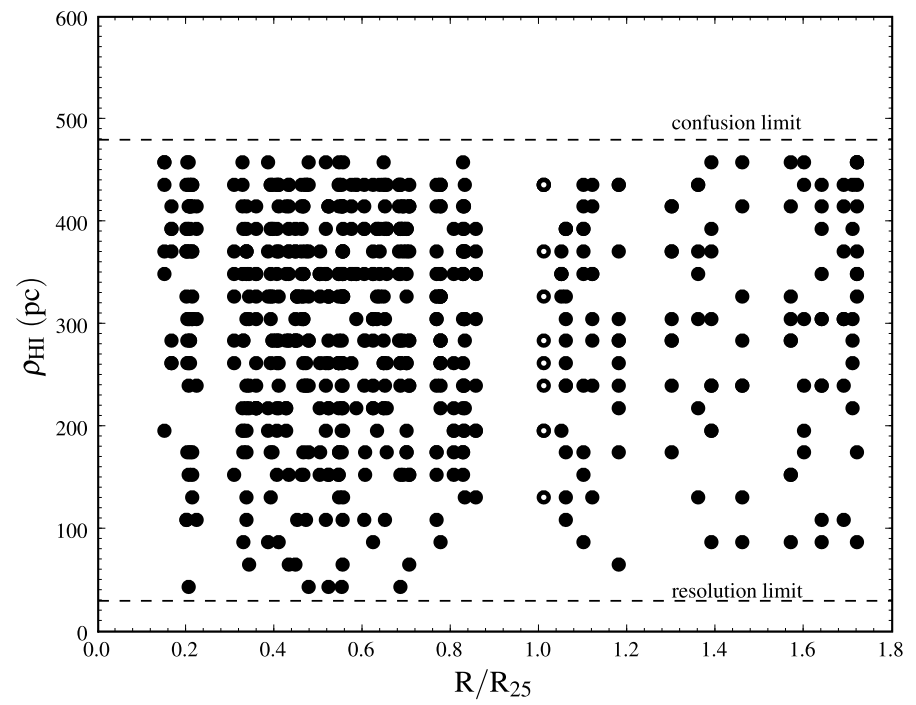

Fig. 5. The distances $\rho_{\mathrm{HI}}$ from the central UV source to individual HI patches are plotted here. Each source can have multiple HI patches associated with it. As an example, $10 \mathrm{HI}$ patches associated with one particular UV source are plotted as open circles here, at $R / R_{25} \sim 1$ (Sources $61 \mathrm{a}-\mathrm{j}$ in Table 3; three sources have an identical separation). At a separation larger than $22^{\prime \prime}$ no HI patches are recorded anymore. In the outer parts fewer HI patches are detected, possibly because of sensitivity issues. Some gaps in the radial coverage can be seen, where no sources were found with our selection method.

is 0.7 . Since photodissociation rates are highly sensitive to the local dust-to-gas ratios, we looked at a number of recent results for M 83.

Different alternative total hydrogen volume densities based on various dust models suggested by Bresolin \& Kennicutt (2002) and Gil de Paz et al. (2007b) are shown in Fig. 9. The different dust models can be found in Fig. 2. The metallicity model based on Bresolin \& Kennicutt (2002) uses

$\log \delta / \delta_{0}=-0.021 R+0.17$.

The slope of this model is relatively shallow. The dual metallicity model in the middle panel uses Eq. (1), except that $\delta / \delta_{0}=0.15$ 
Table 3. HI measurements, incident fluxes and volume densities. The full table is only available in electronic form at the CDS.

\begin{tabular}{ccccccc}
\hline \hline Source No. & $\rho_{\mathrm{HI}}(\mathrm{pc})$ & $N_{\mathrm{HI}}\left(10^{20} \mathrm{~cm}^{-2}\right)$ & $G_{0}$ & $G / G_{\mathrm{bg}}$ & $n\left(\mathrm{~cm}^{-3}\right)$ & Fractional error \\
\hline $1 \mathrm{a}$ & 196 & 1.26 & 1.41 & 2.05 & 120 & 0.8 \\
1b & 349 & 2.77 & 0.45 & 0.65 & 12 & 0.7 \\
1c & 371 & 4.28 & 0.40 & 0.57 & 5 & 0.7 \\
1d & 436 & 6.99 & 0.29 & 0.41 & 1 & 0.9 \\
1e & 458 & 6.69 & 0.26 & 0.38 & 1 & 0.9 \\
1f & 458 & 9.40 & 0.26 & 0.38 & 0.3 & 1.2 \\
2a & 262 & 1.41 & 0.32 & 0.57 & 24 & 0.7 \\
2b & 262 & 2.62 & 0.32 & 0.57 & 10 & 0.7 \\
2c & 284 & 2.77 & 0.27 & 0.49 & 8 & 0.7 \\
2d & 371 & 2.47 & 0.16 & 0.28 & 5 & 0.7 \\
2e & 393 & 2.62 & 0.14 & 0.25 & 4 & 0.7 \\
2f & 393 & 2.92 & 0.14 & 0.25 & 4 & 0.7 \\
2g & 415 & 3.67 & 0.13 & 0.23 & 2 & 0.7 \\
\hline
\end{tabular}

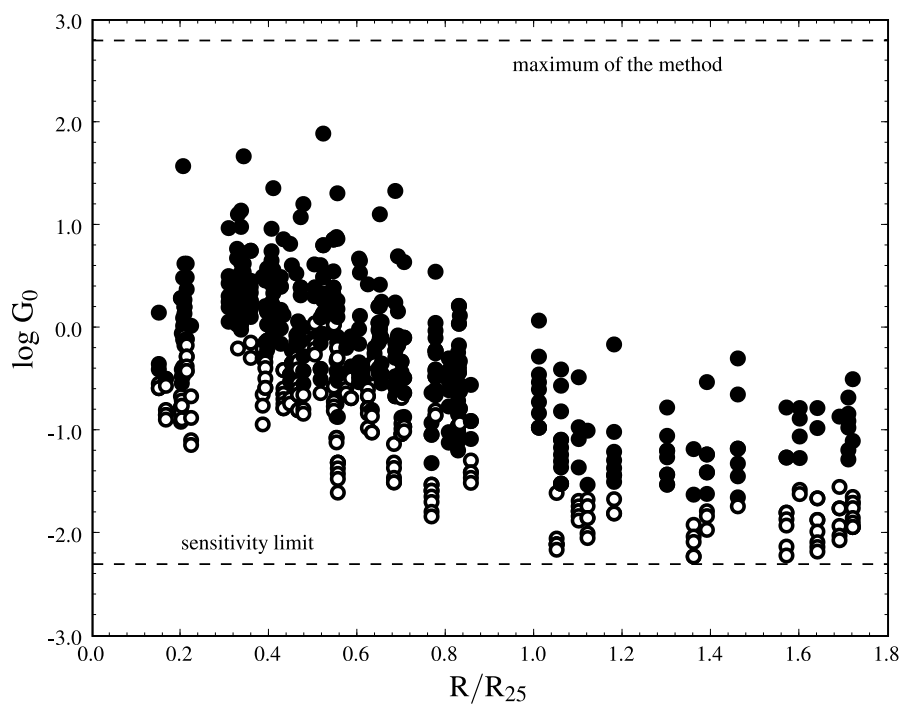

Fig. 6. The incident flux $G_{0}$ is plotted here. Some holes in the radial coverage are visible. The fluxes within $0.2 R_{25}$ seem to be lower. Generally, fluxes are dropping going outward. The maximum of the method consists of the $G_{0}$ that would have resulted from our maximum measured FUV flux, combined with the lowest separation that can be resolved at the given resolution. Sources with a source contrast of less than 0.5 are plotted as open circles.

for all PDRs outside of $R_{25}$, assuming low and constant metallicities in the outer regions of M 83 (Gil de Paz, private communication, 2008). The bottom panel uses

$$
\log \delta / \delta_{0}=-0.100 R+0.85 \text {. }
$$

The slope of the metallicity is relatively steep in this case since a single slope is assumed, with high metallicities inside the optical disk of M 83 and low metallicities outside.

The top panel densities are similar to the ones in Fig. 8, since the underlying dust models are also very similar. The dual metallicity model in the middle panel yields densities in the outer regions that appear to be unusually high. Finally, the densities in the bottom panel are extremely depressed at small galactocentric radii, a result of the high dust content. Insufficient data are available to pick one dust model over the other. We continue our analysis using the high metallicity model of Gil de Paz et al. (2007b), as shown in Fig. 8.

In order to further quantify the volume densities in Fig. 8, socalled box-and-whisker plots are shown in Fig. 10. Since we did not aim to select a complete sample of candidate PDRs, we are

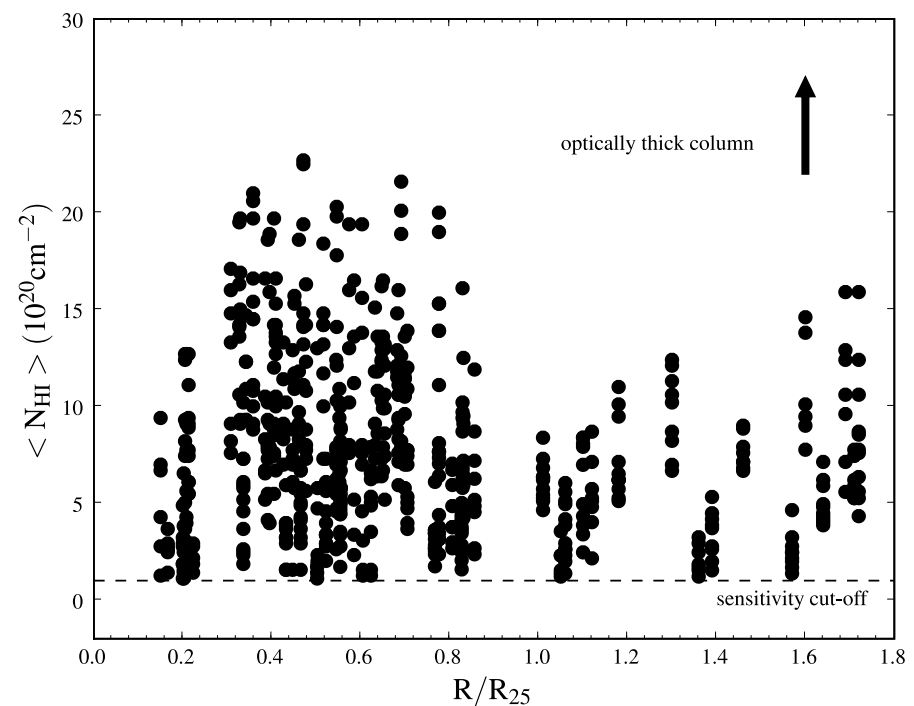

Fig. 7. The (beam averaged) HI column densities are plotted here. Values range from around the sensitivity limit at $1 \times 10^{20} \mathrm{~cm}^{-2}$ to almost $2.5 \times 10^{21} \mathrm{~cm}^{-2}$. Columns are highest within $R_{25}$ and a small upturn can be seen signifying M 83's HI ring at about $1.7 R_{25}$.

mostly interested in the range of volume densities. The galactocentric radius bins in this plot give a good impression of the distribution of the volume densities. There is a wider spread of values in the inner regions of M 83, accompanied by higher density values. The values in the outer regions are more concentrated and the maximum densities are lower. This is mostly due to the narrower spread of HI column densities outside $R_{25}$. Selection effects also limit the range of observed densities.

Our results are summarized in Fig. 11, which is suitable to show the various limiting selection effects. The candidate PDRs at a galactocentric radius larger than $R_{25}$ are plotted as open squares. The Roman numerals I through $\mathrm{V}$ indicate the different limits of our observations:

I. the HI column density upper limit of $5 \times 10^{21} \mathrm{~cm}^{-2}$ at a typical $\delta / \delta_{0}$ of 1.8 , due to the HI column becoming optically thick. However, the actual upper limit of the HI is closer to $2.5 \times 10^{21} \mathrm{~cm}^{-2}$ because of beam smoothing effects. These values occur around a typical $\delta / \delta_{0}$ of 2.4 and are delineated by an extra dashed-dotted line;

II. the HI lower limit of $1 \times 10^{20} \mathrm{~cm}^{-2}$ at a typical $\delta / \delta_{0}$ of 1 . This is a sensitivity limit; 


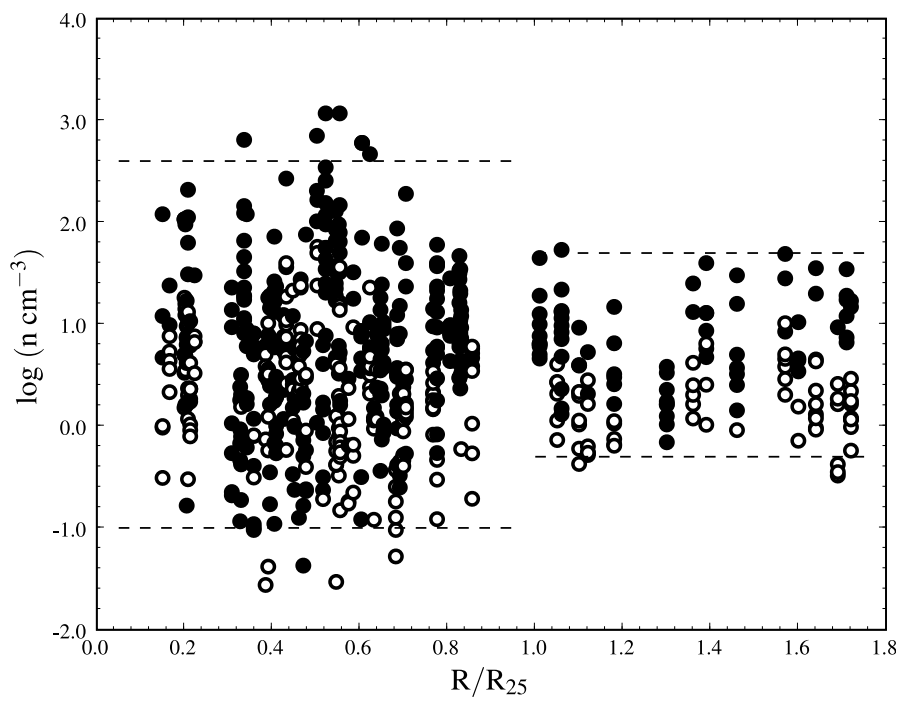

Fig. 8. The total hydrogen volume density is plotted, assuming relatively high metallicity (our preferred dust model, Eq. (1)) outside the optical disk. Closed circles indicate a source contrast higher than 0.5, open circles indicate a lower source contrast. An approximate range of values of $n$ is indicated (dashed lines): $0.1-400 \mathrm{~cm}^{-3}$ inside $R_{25}, 0.5-$ $50 \mathrm{~cm}^{-3}$ outside $R_{25}$.
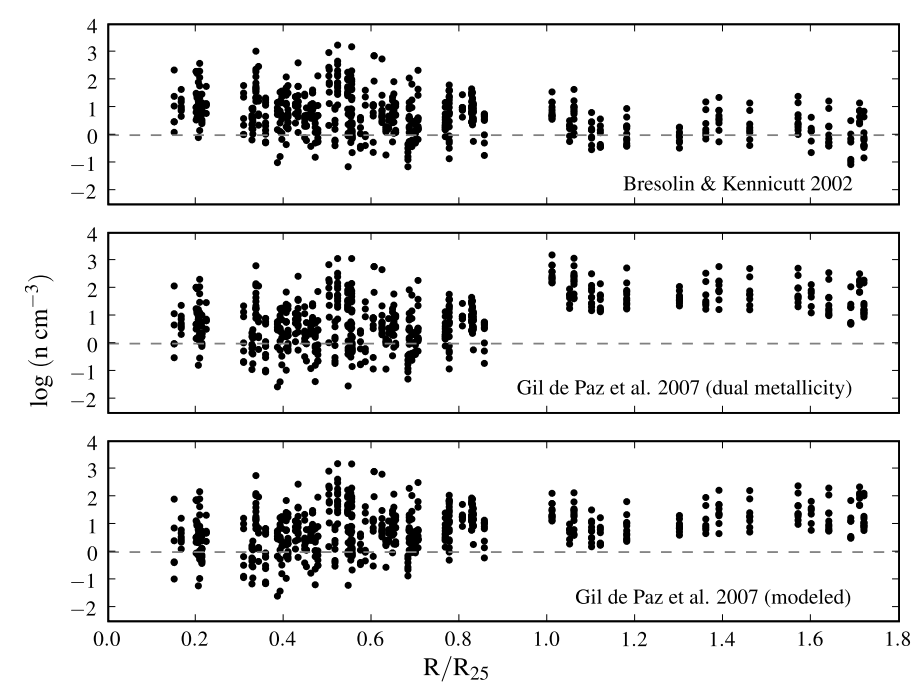

Fig. 9. The total hydrogen volume density is plotted for three dust models derived from Bresolin \& Kennicutt (2002) and Gil de Paz et al. (2007b). The results based on the Bresolin \& Kennicutt (2002) dust model are similar to the one from Gil de Paz et al. (2007b) assuming a high metallicity outside the optical disk (Fig. 8). In the dual metallicity case, the hydrogen densities are relatively high in the outer regions. The single slope fit based on PDR modeling by Gil de Paz et al. (2007b) is steep because of relatively high metallicities near the center of M 83 and low metallicities in the outer regions. Densities are markedly lower in the center in this case.

III. the lowest volume density that we can observe as a consequence of the radio beam diameter and the HI sensitivity limit. For M 83, it is approximately $0.2 \mathrm{~cm}^{-3}$. Points may end up lower because of high metallicities. Ideally, this limit would contain the GMC cloud size. Since this is unknown, the beam diameter is a reasonable substitute;

IV. the minimum $G_{0}(0.005)$ that we can use (at a source contrast as low as $1 \%$ );

V. the maximum $G_{0}(565)$ that we can obtain from the data.

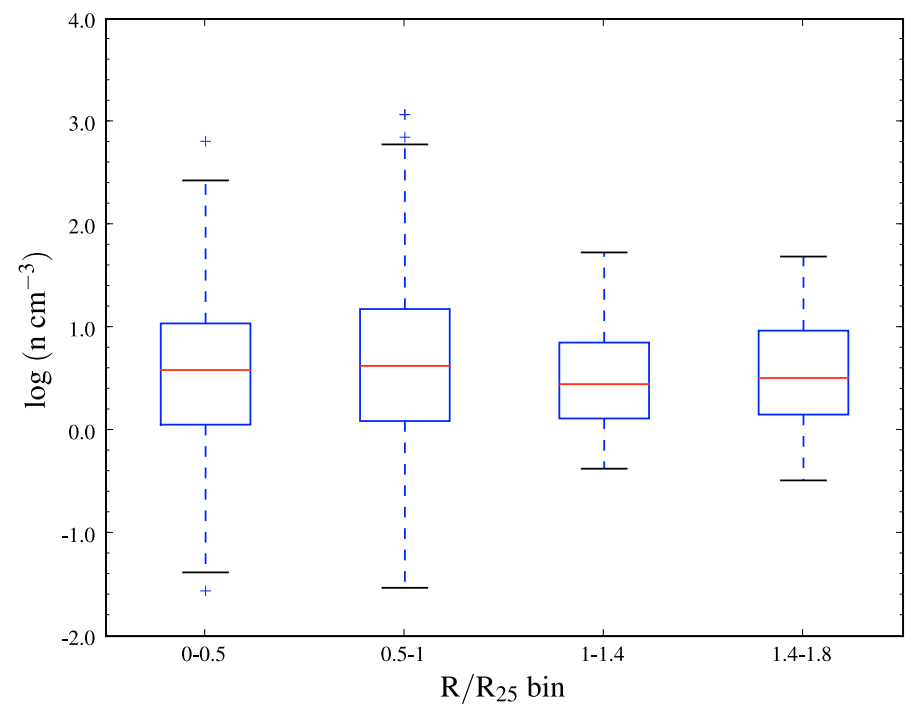

Fig. 10. Box-and-whisker plots for the total hydrogen volume densities in Fig. 8 are displayed, where the data has been divided into four galactocentric radius bins. The boxes span the lower to upper quartiles of the data $(50 \%$ of the datapoints in the bin combined), with a line indicating the median. The so-called "whiskers" show the range of the data (1.5 times the inner quartile range of points), with outliers plotted individually (+). The approximate ranges in Fig. 8 are in good agreement with this. The maximum densities in the outer regions are lower than the ones in the inner regions of M 83 and the range of values is narrower.

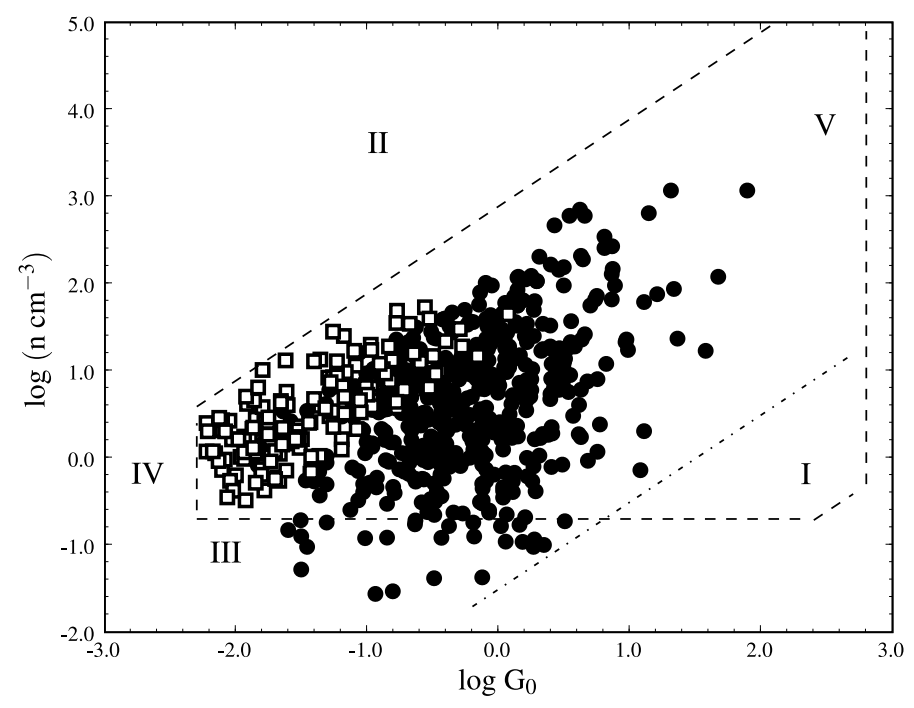

Fig. 11. Our results can be summarized in a plot of $G_{0}$ against $n$. The numerals indicate observational selection effects discussed in the text. In this plot, the open squares indicate regions outside of $R_{25}$.

\section{Discussion and conclusions}

In this section we compare our results to $\mathrm{H}_{2}$ densities based on $\mathrm{CO}$ measurements. Then we discuss background issues, extinction and dust issues. We end with a summary of our findings.

\subsection{CO results comparison}

The study of Rand et al. (1999) is detailed enough to find typical sizes and masses of GMCs in the eastern arm of M 83. These results can be compared to ours with some additional assumptions. Using a $\mathrm{CO}(1-0)$ map with kinematic information, they find CO masses and virial masses of the order of $1 \times 10^{6} M_{\odot}$. 
For example, their source 9 (their Table 4) yields a mass derived from $\mathrm{CO}$ emission of $7.3 \times 10^{6} M_{\odot}$ and $3.6 \times 10^{6} M_{\odot}$ for the virial masses, at a $50-80 \%$ uncertainty. This source corresponds to our FUV source No. 13. Since our method does not yield any information on cloud sizes (we only observe HI on the surface of GMCs), nor on kinetic temperatures in these clouds, we will take typical values of our measured volume densities and compare densities using a typical GMC radius of $75 \mathrm{pc}$ for a spherical cloud. $n=2 n_{\mathrm{H}_{2}}$ inside the GMC and a typical density near our source No. 13 is $20 \mathrm{~cm}^{-3}$, or $n_{\mathrm{H}_{2}}=10 \mathrm{~cm}^{-3} .7 .3 \times 10^{6} M_{\odot}$ is equivalent to about $n_{\mathrm{H}_{2}}=85 \mathrm{~cm}^{-3}$, which is in reasonable agreement with our results considering the uncertainties in both results (70-80\%). More recent unpublished data (Lord, private communication, 2008) may be quantitatively different and more detailed, but does not yield substantially different results at the resolution of the data that were used in this paper.

One of the key differences between M81 and M 83 is the abundance of $\mathrm{CO}$ emission. While $\mathrm{CO}$ is extremely faint and hard to detect in M 81 (e.g. Casasola et al. 2007), M 83 displays bright $\mathrm{CO}$ features. The method we used here to find hydrogen densities (and molecular hydrogen densities in GMCs) does not yield any morphological information, since we are observing $\mathrm{HI}$ on the surface of PDRs. We can note, however, that the size and scale we assume for our candidate PDRs (the range of values of $\left.\rho_{\mathrm{HI}}\right)$ is consistent with findings using CO. Since we did not find suitable candidate PDRs in the inner kiloparsec of M 83, we cannot compare our results with for example the results of Wall et al. (1993), although the densities we find match their low-density component. As to the truncation of the molecular disk that was mentioned by Crosthwaite et al. (2002), we do see a difference between the inner and outer disk of M 83, but it does not seem to be that significant in our results. No differences are seen in candidate PDRs in arm or inter-arm regions, insofar as the large scatter in our results allows us to draw this conclusion. We do not find more than a handful of regions with the densities indicated by Hosokawa \& Inutsuka (2007), although the expected sizes of our candidate PDRs are the same. Finally, the hydrogen densities presented here are similar to the M 81 results but extend to higher maximum values. This is consistent with the brighter $\mathrm{CO}$ emission in $\mathrm{M} 83$, since the higher $\mathrm{H}_{2}$ densities will lead to a greater degree of excitation of the $\mathrm{CO}$ molecules. Alternatively, this could also indicate a higher fraction of $\mathrm{CO}$ molecules in M 83's clouds (e.g. Casasola et al. 2007).

\subsection{Background levels, extinction and dust}

The FUV fluxes seem to be higher in the inner parts of M 83 and decreasing (on average) going outward, as is shown in Fig. 4. These higher fluxes in the inner parts could be caused by brighter sources, and/or a larger number of sources. Since the individual sources are unresolved, no firm conclusions can be drawn here. Outside $R_{25}$ the measured fluxes are roughly constant. At the same time, the abundances of PDR-produced HI surrounding these FUV sources (Fig. 7 - note that these are individual measurements, not annular averages) seem to follow a similar trend, consistent with its connection to the photodissociating UV radiation. The FUV source contrast does not vary with galactocentric radius, so while both source and background radiation field decrease in intensity towards the outer regions of M 83, their relative strength does not change.

Another factor that could influence the FUV radiation is galactic foreground extinction. The higher the applied foreground extinction, the higher the resulting total hydrogen volume density. The $0.52 \mathrm{mag}$ extinction (Schlegel et al. 1998) towards M 83 is significantly less than the 1.37 mag we used towards M 81 in Heiner et al. (2008). The Schlegel et al. (1998) extinction correction for M 81 would be 0.58 , significantly lowering the total hydrogen volume densities. We find a wider range of values in M 83, including higher gas densities. We therefore expect M 83 to harbor more gas than M 81. The M 81 and M 83 results are hard to compare directly because the extinction corrections were based on different sources in the literature. We intend to compare the two galaxies, together with similar M33 results, more quantitatively in a future paper, with consistent extinction corrections.

Our results are also sensitive to the dust-to-gas ratio. The slope of M 83's metallicity is relatively shallow. Our preferred dust model assumes that metallicities in the outer parts of M 83 remain high. Lower metallicities would result in higher gas densities. Our results in these regions are therefore most likely lower limits and much more gas could be present. The recent results by Gil de Paz et al. (2007b) are ambiguous in the sense that the authors provide high metallicity and low metallicity results, depending on the adopted model. In the high metallicity scenario, the slope of M 83's metallicity remains shallow out to large galactocentric radius. The low metallicity scenario is accompanied by a sharp drop in metallicity starting at approximately $R_{25}$.

Smith et al. (2000) found a similar range of gas densities in M101 using basically the same method with additional extinction corrections. Their candidate PDRs were all within M101's $R_{25}$, but the FUV luminosities within that range are similar. As in our previous M 81 results, no internal extinction correction was applied. If any such correction were applied, it should scale with the dust-to-gas ratio. This means that the extinction would decrease going outward. The FUV fluxes would be even higher in the center of M 83 (leading to higher total hydrogen volume densities), while the fluxes in the outer regions of M 83 would not be affected much.

\subsection{Summary}

In summary, we have investigated atomic hydrogen found in candidate PDRs in M 83 and used the physics of these PDRs to derive total hydrogen volume densities. We carefully considered the contribution of observational selection effects to our results. We find a range of densities: $0.1-400 \mathrm{~cm}^{-3}$ inside $R_{25}$, $0.5-50 \mathrm{~cm}^{-3}$ outside $R_{25}$, based on measurements of HI believed to be produced in large scale PDRs in M 83. The higher GMC volume densities which we find within $R_{25}$ correlate well with the presence of bright $\mathrm{CO}(1-0)$ emission there. This points to enhanced collisional excitation as one reason for the $\mathrm{CO}$ emission in these GMCs. We note that this is also consistent with our results in M 81, where we find little evidence for high GMC volume densities, and for which the $\mathrm{CO}(1-0)$ emission is faint.

Our measurements go out to a galactocentric radius of $13 \mathrm{kpc}$ (deprojected). Our study used the tilted ring model from Rogstad et al. (1974) to get proper galactocentric radii outside the M 83 optical disk. Our results are notably sensitive to the local dustto-gas ratio, especially since the metallicity in the outer regions remains uncertain.

Acknowledgements. The authors are grateful to Fabian Walter, Elias Brinks and the THINGS team for providing us with M 83 radio data as well as for engaging in useful discussions. We would also like to thank Gerhardt Meurer for his help and useful discussions, as well as David Thilker, Armando Gil de Paz and Steven Lord. We thank the anonymous referee for providing suggestions that 
improved the clarity of this work. J.S.H. acknowledges the support of a Graduate Research Assistantship provided by the STScI Director's Discretionary Research Fund.

\section{References}

Allen, R. J. 2004, in Penetrating Bars Through Masks of Cosmic Dust, ed. D. L. Block, I. Puerari, K. C. Freeman, R. Groess, \& E. K. Block (Astrophysics and Space Science Library), 319, 731

Allen, R. J., Atherton, P. D., \& Tilanus, R. P. J. 1986, Nature, 319, 296

Allen, R. J., Knapen, J. H., Bohlin, R., \& Stecher, T. P. 1997, ApJ, 487, 171

Allen, R. J., Heaton, H. I., \& Kaufman, M. J. 2004, ApJ, 608, 314

Allende Prieto, C., Lambert, D. L., \& Asplund, M. 2001, ApJ, 556, L63

Boissier, S., Gil de Paz, A., Madore, B. F., et al. 2005, ApJ, 619, L83

Bresolin, F., \& Kennicutt, Jr., R. C. 2002, ApJ, 572, 838

Casasola, V., Combes, F., Bettoni, D., \& Galletta, G. 2007, A\&A, 473, 771

Crosthwaite, L. P., Turner, J. L., Buchholz, L., Ho, P. T. P., \& Martin, R. N. 2002 , AJ, 123, 1892

Ekers, R. D., Allen, R. J., \& Luyten, J. R. 1973, A\&A, 27, 77

Gil de Paz, A., Boissier, S., Madore, B. F., et al. 2007a, ApJS, 173, 185

Gil de Paz, A., Madore, B. F., Boissier, S., et al. 2007b, ApJ, 661, 115

Heiner, J. S., Allen, R. J., Emonts, B. H. C., \& van der Kruit, P. C. 2008, ApJ, 673,798
Hosokawa, T., \& Inutsuka, S.-i. 2007, ApJ, 664, 363

Issa, M. R., MacLaren, I., \& Wolfendale, A. W. 1990, A\&A, 236, 237

Lasker, B. M., Lattanzi, M. G., McLean, B. J., et al. 2008, AJ, 136, 735

Lord, S. D., \& Kenney, J. D. P. 1991, ApJ, 381, 130

Lundgren, A. A., Wiklind, T., Olofsson, H., \& Rydbeck, G. 2004, A\&A, 413, 505

Rand, R. J., Lord, S. D., \& Higdon, J. L. 1999, ApJ, 513, 720

Rogstad, D. H., Lockhart, I. A., \& Wright, M. C. H. 1974, ApJ, 193, 309

Schlegel, D. J., Finkbeiner, D. P., \& Davis, M. 1998, ApJ, 500, 525

Smith, D. A., Allen, R. J., Bohlin, R. C., Nicholson, N., \& Stecher, T. P. 2000, ApJ, 538, 608

Thilker, D. A., Bianchi, L., Boissier, S., et al. 2005, ApJ, 619, L79

Thim, F., Tammann, G. A., Saha, A., et al. 2003, ApJ, 590, 256

Tilanus, R. P. J., \& Allen, R. J. 1993, A\&A, 274, 707

van der Hulst, J. M., Terlouw, J. P., Begeman, K. G., Zwitser, W., \& Roelfsema, P. R. 1992, in Astronomical Data Analysis Software and Systems I, ed. D. M. Worrall, C. Biemesderfer, \& J. Barnes, ASP Conf. Ser., 25, 131

Vila-Costas, M. B., \& Edmunds, M. G. 1992, MNRAS, 259, 121

Vogelaar, M. G. R., \& Terlouw, J. P. 2001, in Astronomical Data Analysis Software and Systems X, ed. F. R. Harnden, Jr., F. A. Primini, \& H. E. Payne, ASP Conf. Ser., 238, 358

Vogler, A., Madden, S. C., Beck, R., et al. 2005, A\&A, 441, 491

Wall, W. F., Jaffe, D. T., Bash, F. N., et al. 1993, ApJ, 414, 98

Zaritsky, D., \& Christlein, D. 2007, AJ, 134, 135 
J. S. Heiner et al.: The volume densities of giant molecular clouds in M 83, Online Material p 1
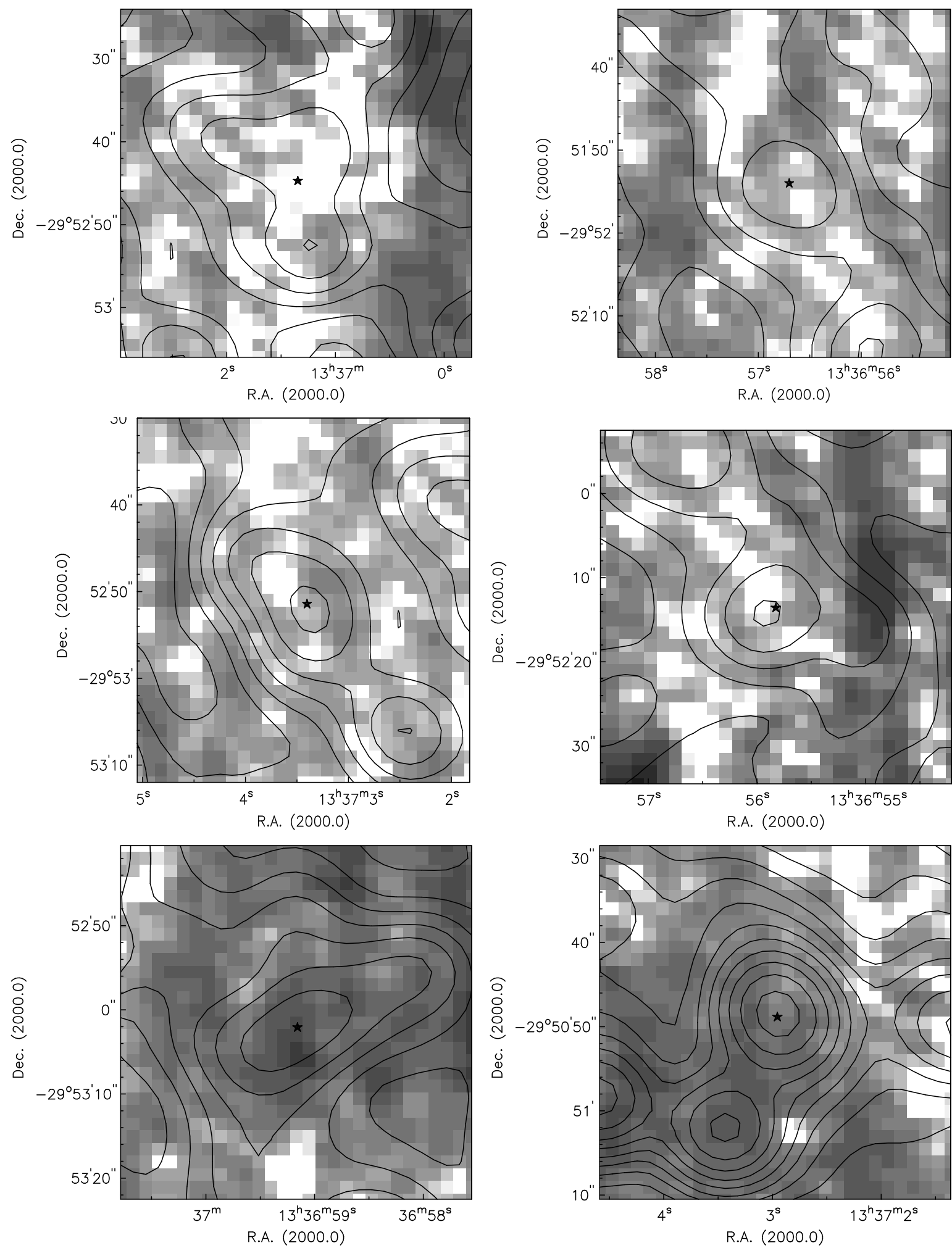

Fig. 3. 
J. S. Heiner et al.: The volume densities of giant molecular clouds in M 83, Online Material p 2
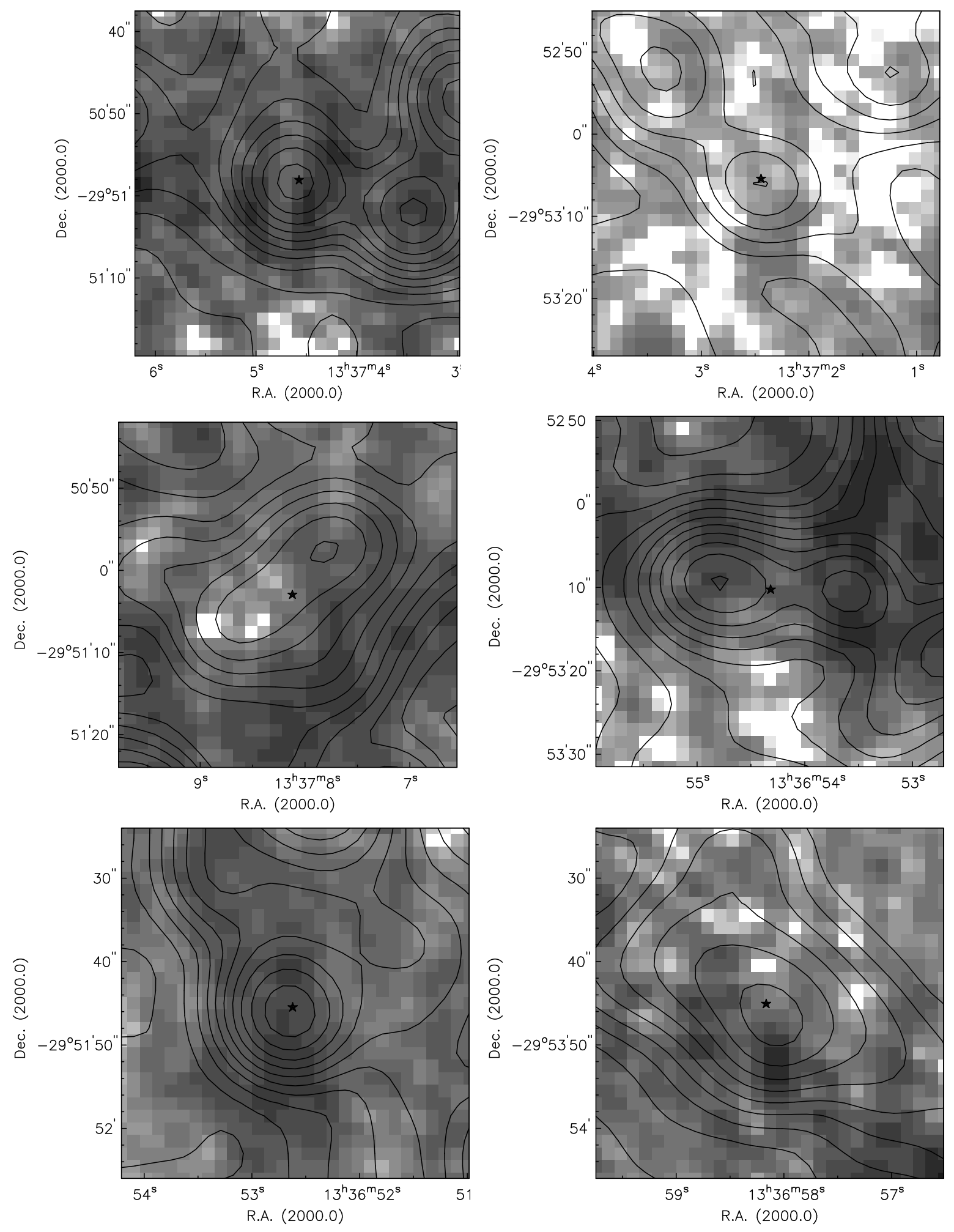

Fig. 3. continued. 
J. S. Heiner et al.: The volume densities of giant molecular clouds in M 83, Online Material p 3
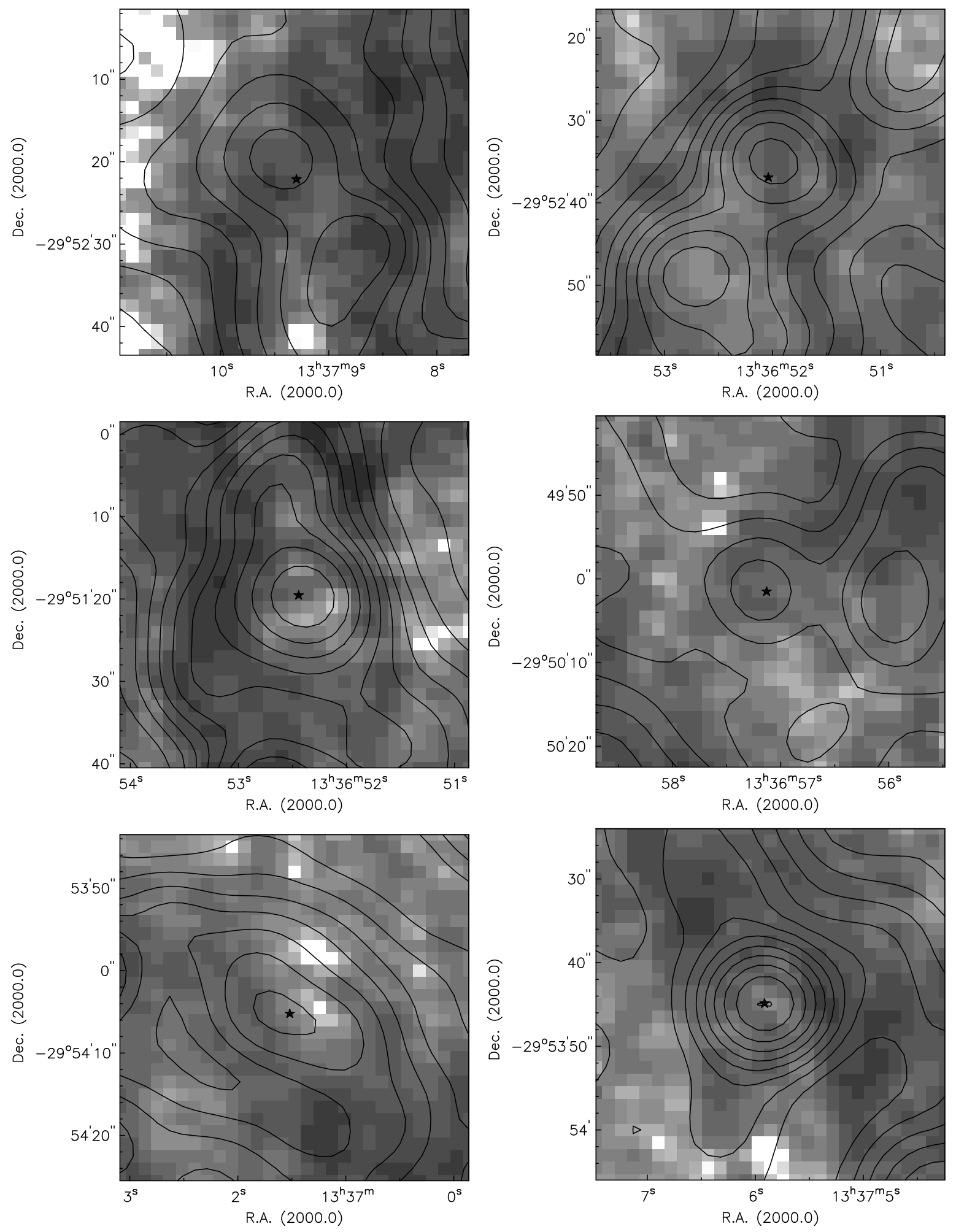

Fig. 3. continued. 
J. S. Heiner et al.: The volume densities of giant molecular clouds in M 83, Online Material p 4
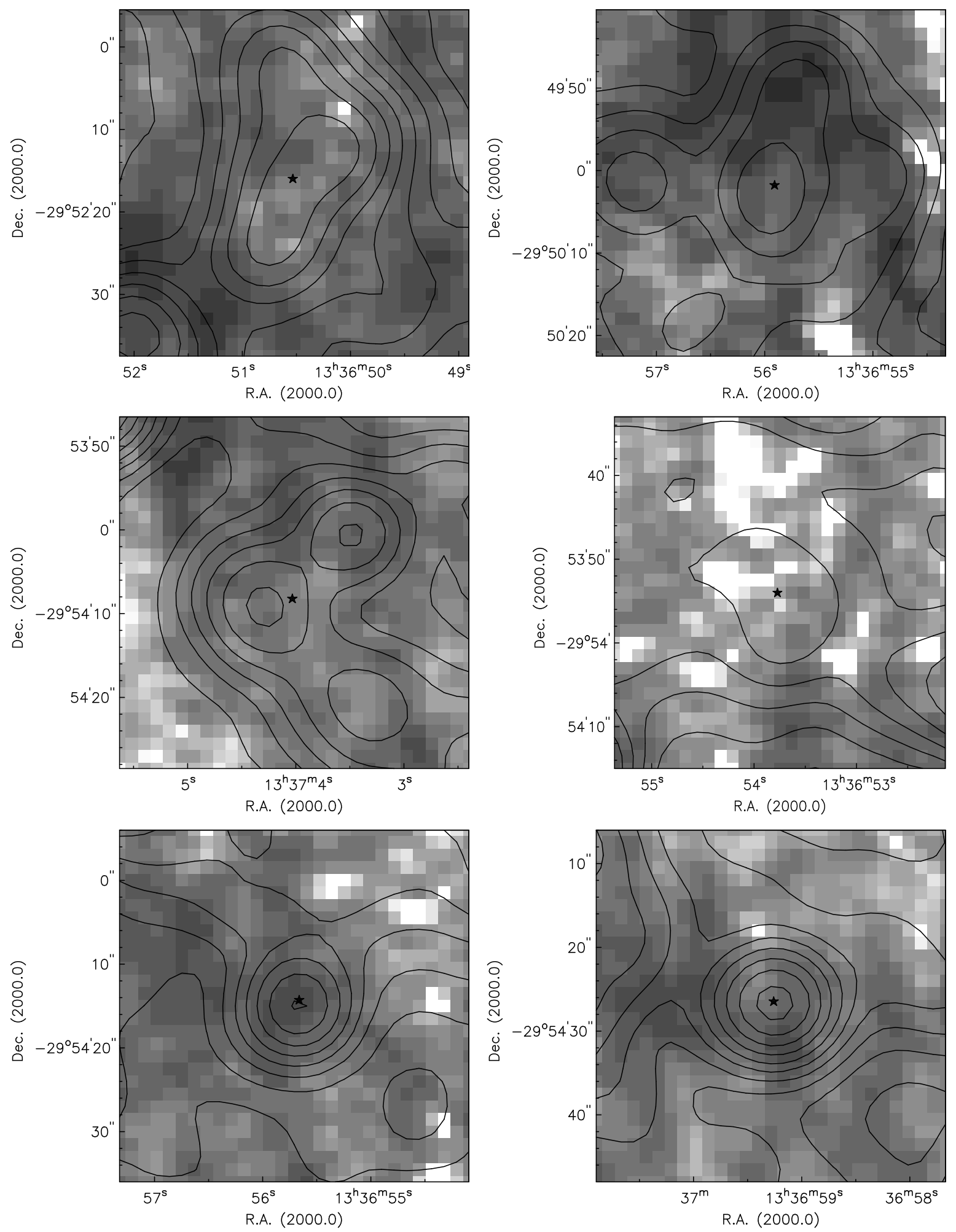

Fig. 3. continued. 
J. S. Heiner et al.: The volume densities of giant molecular clouds in M 83, Online Material p 5
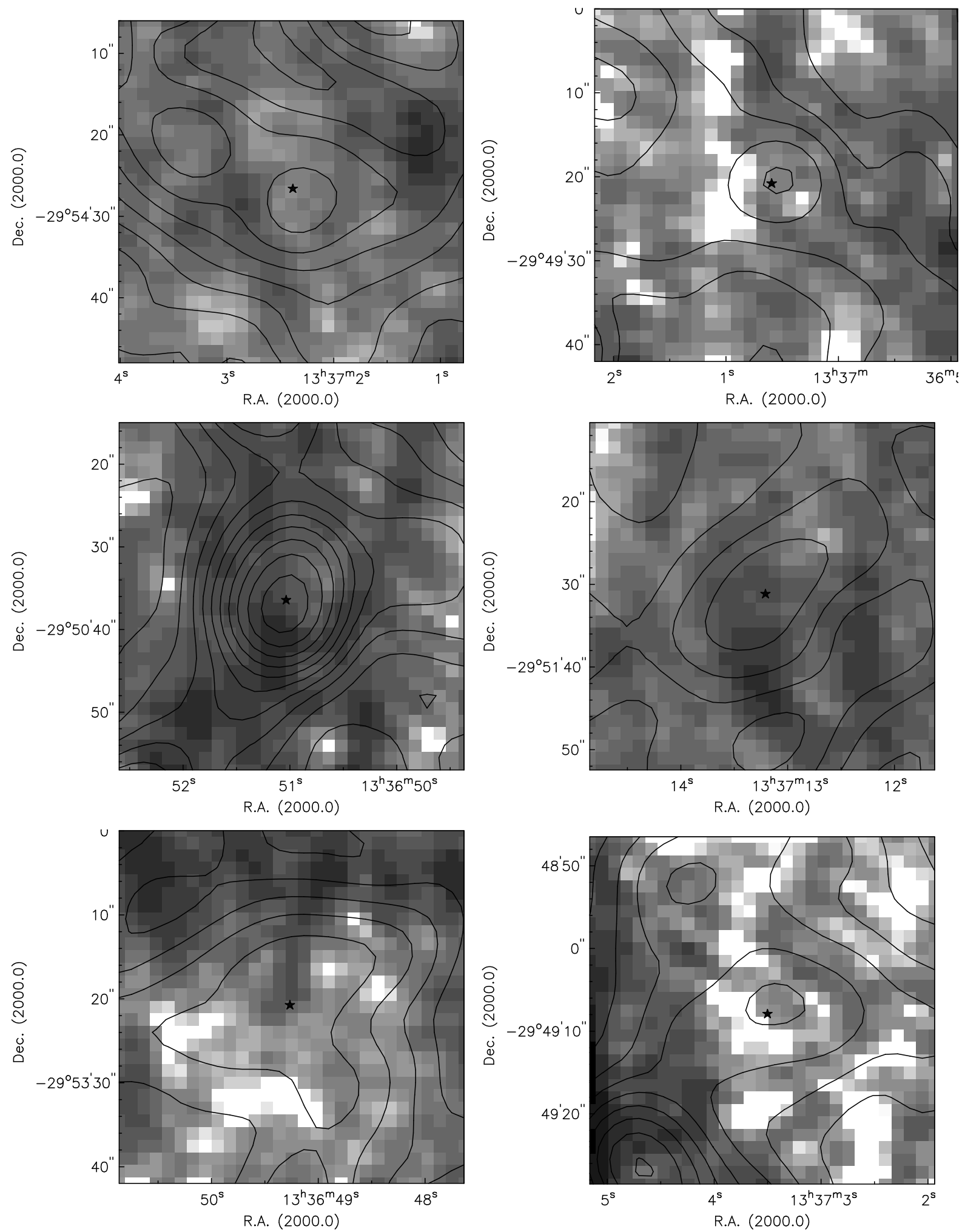

Fig. 3. continued. 
J. S. Heiner et al.: The volume densities of giant molecular clouds in M 83, Online Material p 6
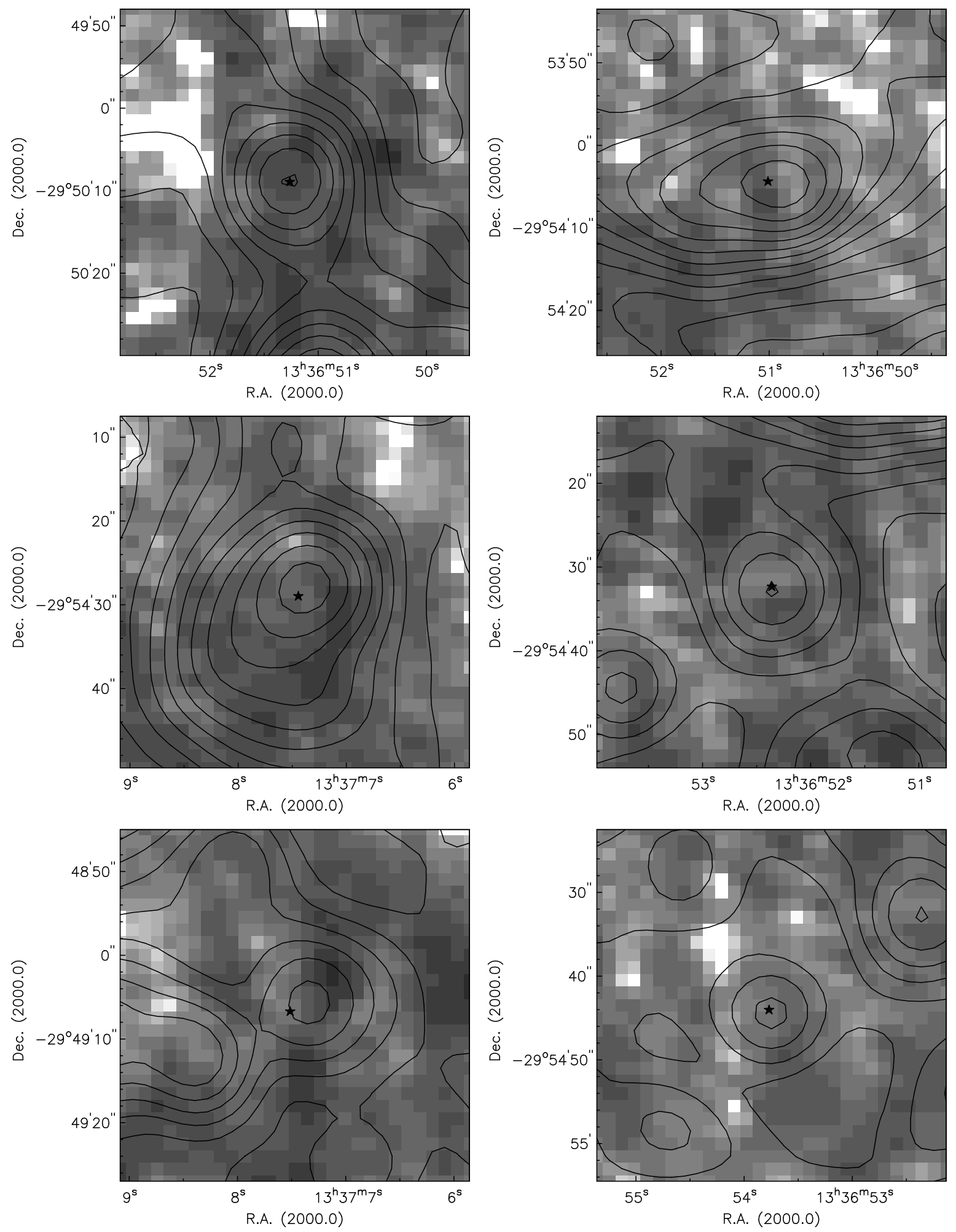

Fig. 3. continued. 
J. S. Heiner et al.: The volume densities of giant molecular clouds in M 83, Online Material $p 7$
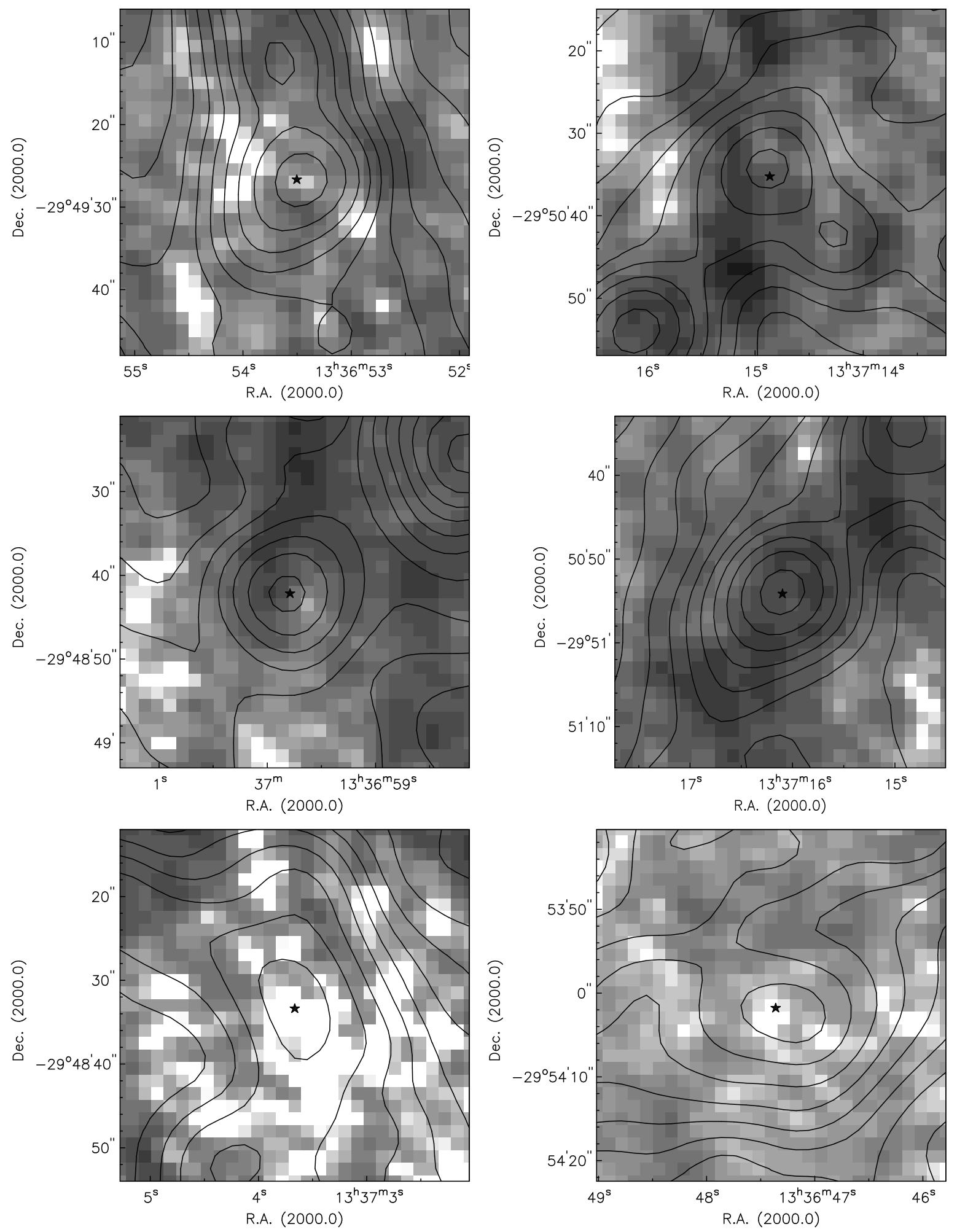

Fig. 3. continued. 
J. S. Heiner et al.: The volume densities of giant molecular clouds in M 83, Online Material $p 8$
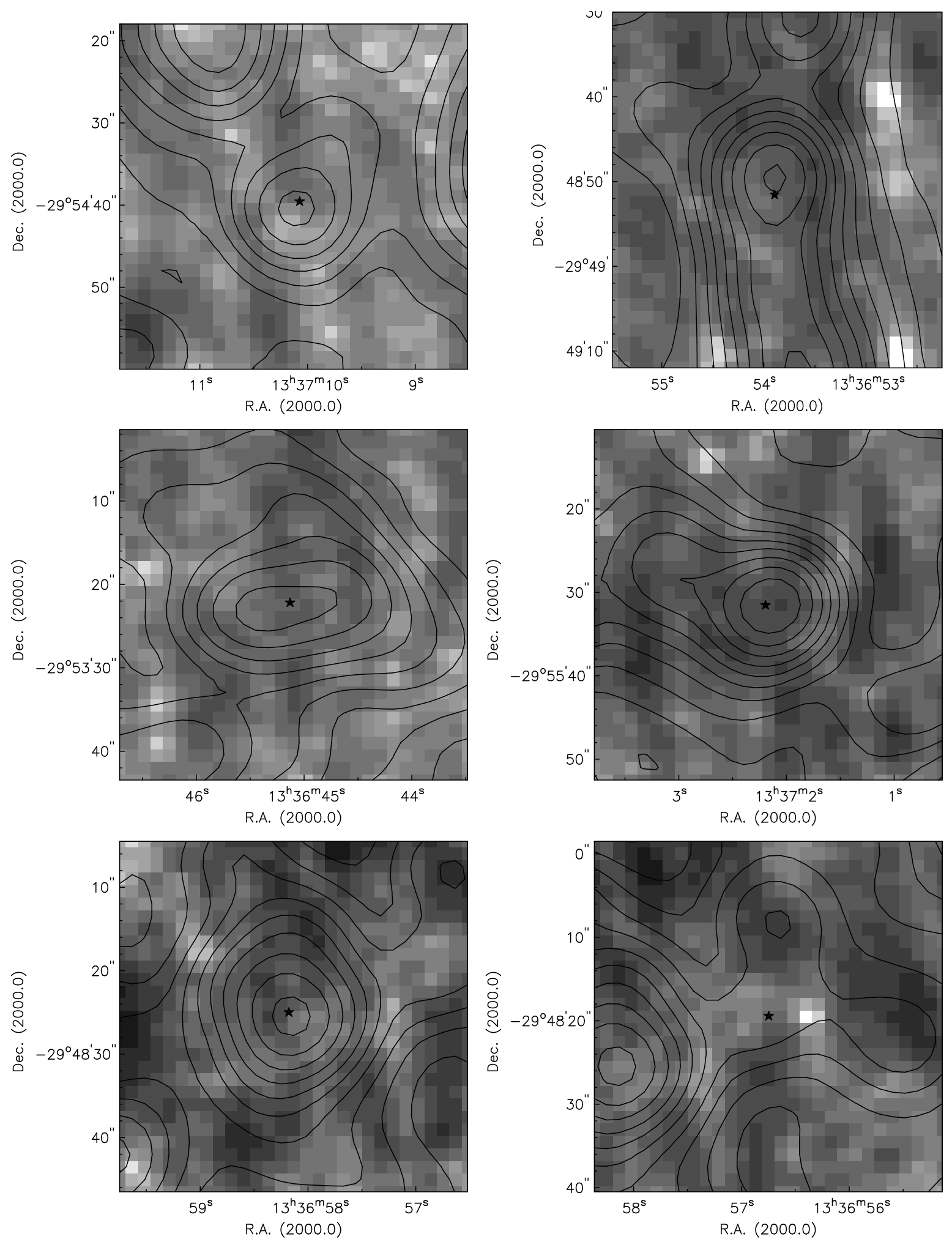

Fig. 3. continued. 
J. S. Heiner et al.: The volume densities of giant molecular clouds in M 83, Online Material p 9
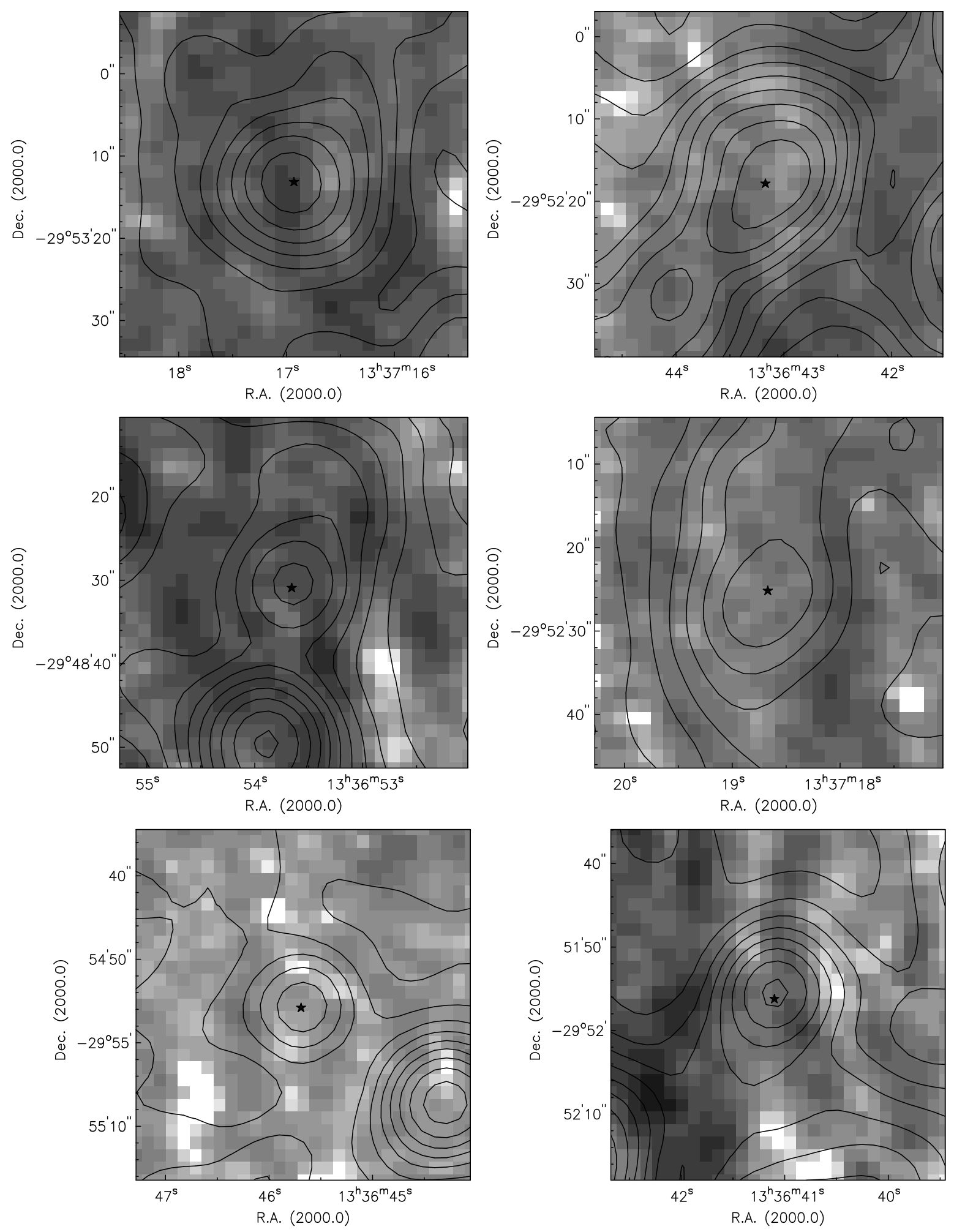

Fig. 3. continued. 
J. S. Heiner et al.: The volume densities of giant molecular clouds in M 83, Online Material p 10
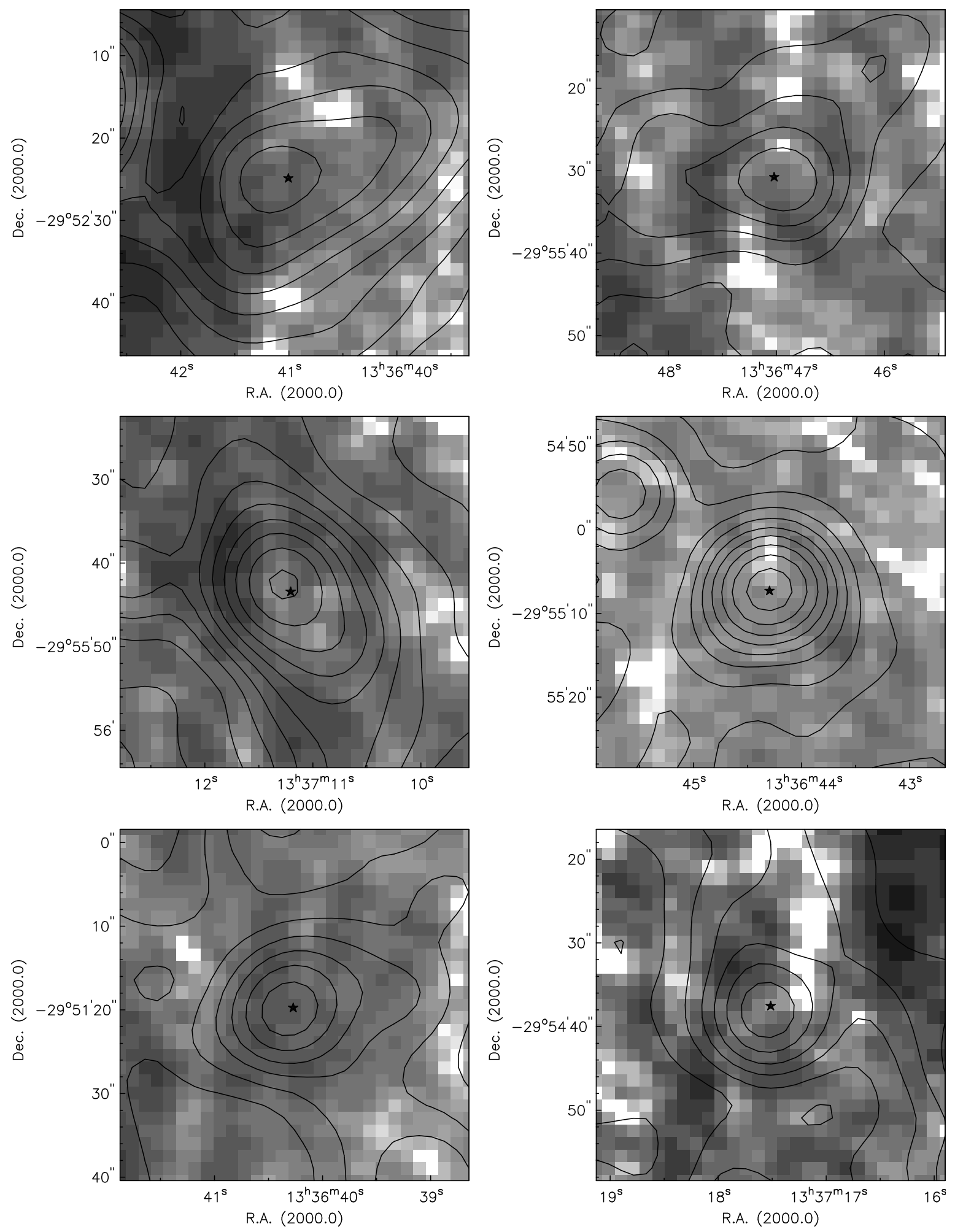

Fig. 3. continued. 
J. S. Heiner et al.: The volume densities of giant molecular clouds in M 83, Online Material $p 11$
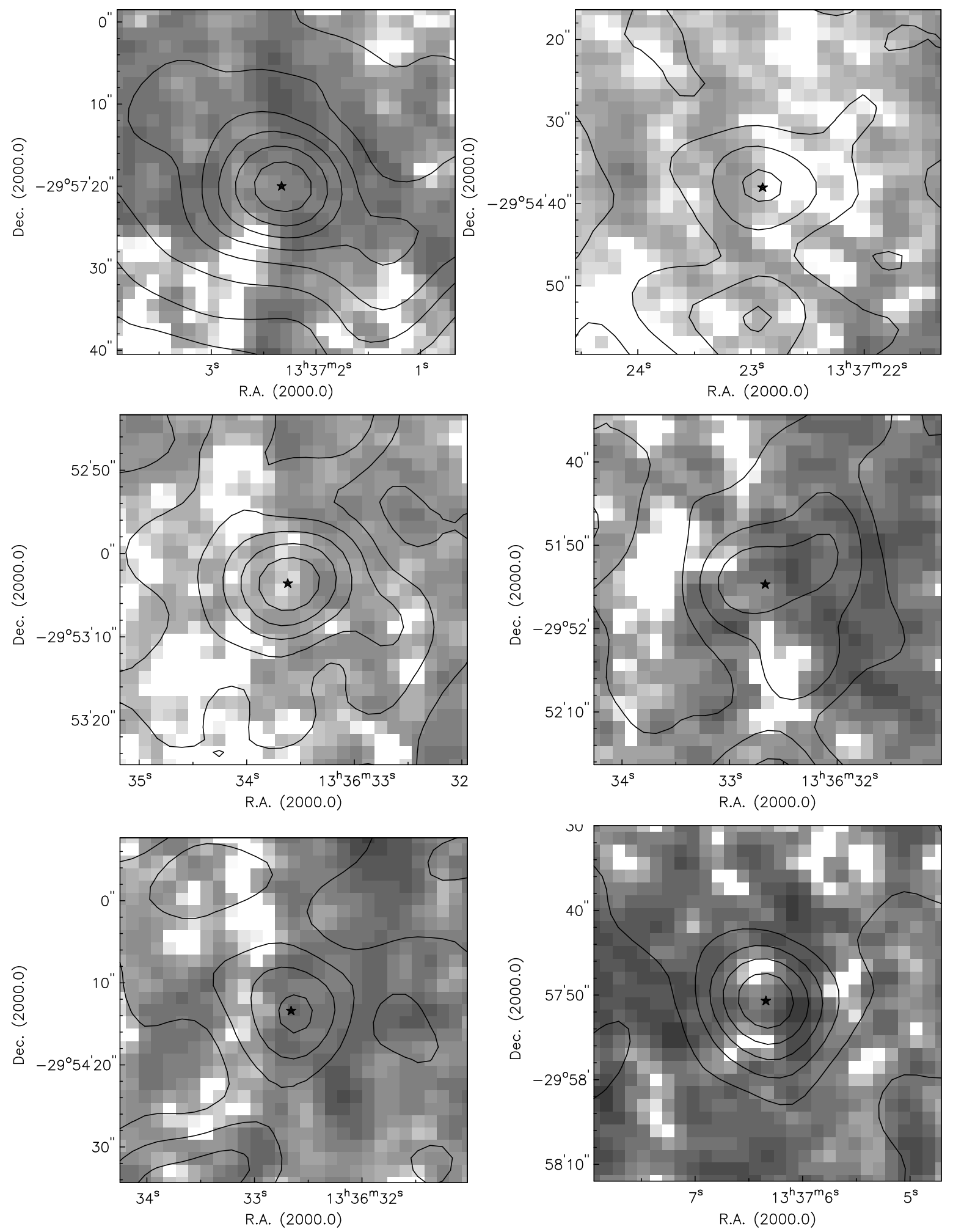

Fig. 3. continued. 
J. S. Heiner et al.: The volume densities of giant molecular clouds in M 83, Online Material p 12
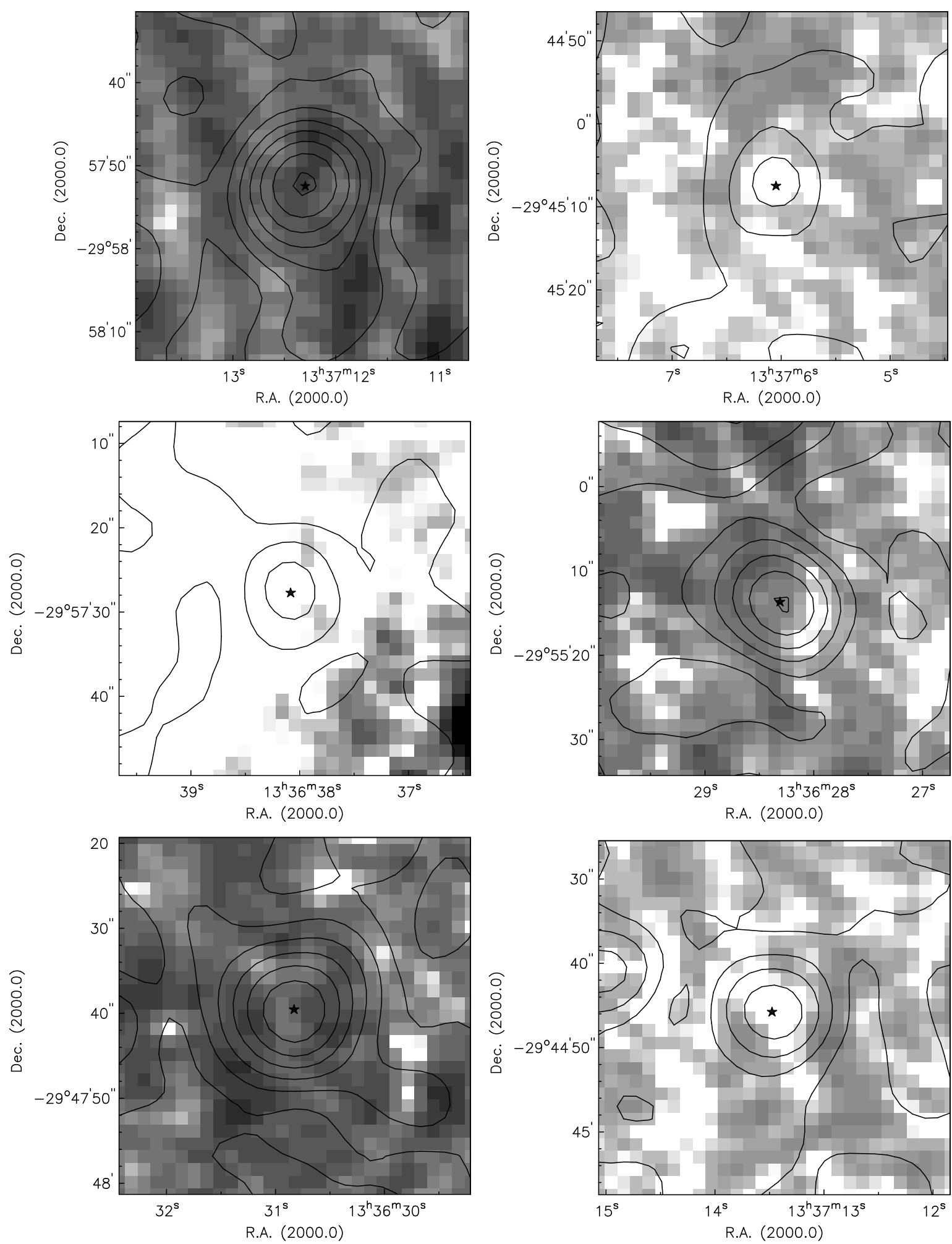

Fig. 3. continued. 
J. S. Heiner et al.: The volume densities of giant molecular clouds in M 83, Online Material $p 13$
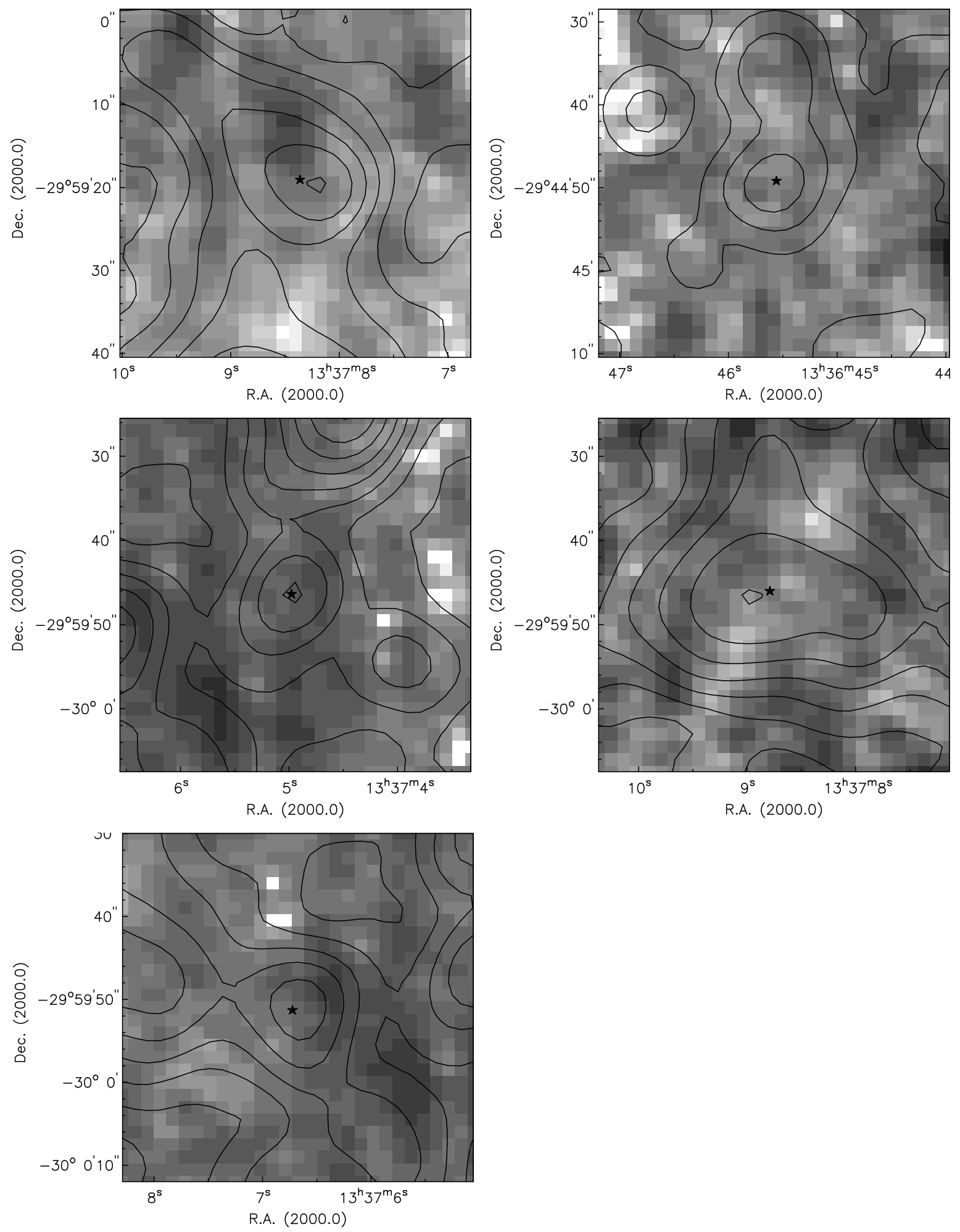

Fig. 3. continued. 